\title{
Morphological Hit-or-Miss Transformation for Shape Recognition ${ }^{1}$
}

\author{
Dongming Zhao* and David G. Daut $\dagger$ \\ *Department of Electrical and Computer Engineering, University of Michigan-Dearborn, Dearborn, Michigan 48128-1491; and \\ $\dagger$ Department of Electrical and Computer Engineering, Rutgers University, P.O. Box 1390, Piscataway, New Jersey 08855-1390
}

Received June 8, 1990; accepted March 4, 1991

In this paper, the morphological hit-or-miss transformation is analyzed for use in the recognition of both perfect shapes and imperfect shapes. Shape recognition is achieved by locating the objects to be recognized within the image. The shape recognition problem is discussed in the following two aspects. First, a theorem is presented in which the hit-or-miss operations employ the structuring elements $\partial A$ and $\partial\left(W \cap A^{\mathrm{c}}\right)$, instead of using sets $A$ and $\left(W \cap A^{\mathrm{c}}\right.$ ). Here $A$ is the object to be recognized, $W$ is a window containing $A, \partial A$ is the boundary of $A$, and $\partial\left(W \cap A^{\mathrm{c}}\right)$ is the boundary of $\left(W \cap A^{\mathrm{c}}\right)$. Second, the recognition of imperfect shapes due to indeterminate variation of an object shape is studied here. Our method employs a priori known shape information as a basis for structuring elements and constructs structuring elements which are then used in a hit-or-miss transformation to find the location of the shape to be recognized. Each occurrence of a target shape is represented either by one point or by a small cluster of points within a calculated range according to the associated structuring elements. Finally, we present the hit-or-miss operation without window restrictions as a technique for recognizing both perfect and imperfect shapes, thereby making the method more flexible. 1991 Academic Press, Inc.

\section{INTRODUCTION}

Shape recognition using mathematical morphology is one of the fundamental image analysis methods [7-12]. There are many important applications in machine vision and automated inspection where the shape of an object is required to be recognized [15-19]. The morphological hit-or-miss transformation is a special tool used to locate known objects. In this situation the problem of shape recognition is equated to that of detecting occurrences of an object shape within an image [3]. In the case of binary images, the problem of shape recognition is simpler since the image function $f(x, y)$ describing the image defined on a 2-D domain takes on only binary values ( 0 or 1$)$ that are specifically defined on $Z^{2}$. In this paper, the use and discussion of set operations are based on the discrete-valued domain $Z^{2}$. The shapes and images are assumed to be the

\footnotetext{
${ }^{1}$ This research was supported, in part, by CAIP Center, Rutgers University, NJ.
}

form of a digital representation. The sets are compact in general; that is, the sets are closed and bounded $[1,2,4]$.

The shape recognition (or, shape matching) process can be generally described by an operation called the hitor-miss transformation $[1, \mathrm{pp} .39-40 ; 6]$. The hit-or-miss operation is defined upon image $I$ as $[I \ominus A] \cap\left[I^{\mathrm{c}} \ominus B\right]$, where $(A, B) \subseteq G$ and $G$ is a general structuring element. Here $\ominus$ is the notation for erosion, where $X \ominus Y=$ $\{z: Y+z \subseteq X\}=\bigcap_{y \in Y} X_{-y}$. The symbol $X_{p}$ is the transformation of $X$ by $p$, that is, $X_{p}=\{z=a+p: a \in X\}$. The image $I$ is eroded by a structuring element (SE) $A$, and the complement of the image, $I^{\mathrm{c}}$, is eroded by an SE $B$. The results of the two erosion operations are then ANDed producing an output set. Given that $A$ represents a shape to be recognized within $I$, and $B$ is a windowed complement of $A$, then the resulting point set of a hit-or-miss transformation consists of points where each point indicates one occurrence of $A$ within $I$. The function of window $W$ is to guarantee that the range of the hit-or-miss operation on shape $A$ does not overlap any portion of other objects that may be within the image. This process can be summarized as [3]: the shape $A$ in window $W$ occurs in the image $I$ at, and only at, the locations represented by set $L$, where $L=[I \ominus A] \cap\left[I^{\mathfrak{c}} \ominus\left(W \cap A^{\mathfrak{c}}\right)\right]$.

Shapes having points within $I \ominus A$ do not have any points which will fall into $I^{\mathrm{c}} \ominus\left(W \cap A^{\mathrm{c}}\right)$, and vice versa, except of course for shape $A$. Therefore, the intersection of the results of the above two erosions will leave those points corresponding to shape $\Lambda$ only. In many situations, the digital images obtained are not perfect; that is, the shapes of objects in the acquired field of view may not be represented exactly the same as the ideal original shapes used for generating the structuring elements. In the case of imperfect images, a pattern is taken as a family of sets $\{A(\gamma)\}, \gamma \in \Gamma$, along with the convention that the pattern $\{A(\gamma)\}$ occurs at $z$ if, and only if, $A_{z}(\gamma)$ occurs in the image for some $\gamma \in \Gamma$. This concept is formalized as [3]: the pattern $\{A(\gamma)\}$ occurs in the image I at the locations represented by set $L$, where $L=\bigcup_{\gamma \in \Gamma}[I \theta$ $A(\gamma)] \cap\left[I^{\mathrm{c}} \ominus\left(W \cap A^{\mathrm{c}}(\gamma)\right)\right]$.

The theorems in [3] are well established; however, redundancy is inherent in the hit-or-miss process. Redun- 
dancy is present in the form of unnecessary intersections of frame shifts subject to all points in set $A$ and set ( $W \cap$ $A^{\mathrm{c}}$ ). It has been suggested that $A$ and $\left(W \cap A^{\mathrm{c}}\right.$ ) be replaced by a minimal set representation [5]. We consider that since the image is in binary format, it is not necessary to represent an object by all points within the object. A subset of the set of object points should be able to describe the characteristics of the binary set, or object. It would be reasonable to think of finding a subset $\delta\{A\} \subset A$ such that by substituting $\delta\{A\}$ for $A$ as a structuring element the recognition process could proceed as if $A$ were used. By the same reasoning, a subset $\delta\left\{W \cap A^{c}\right\} \subset(W \cap$ $A^{\mathrm{c}}$ ) can be used to substitute for the set $\left(W \cap A^{\mathrm{c}}\right)$ as the structuring element for the complement of the image. By substituting subsets of the shapes, we can considerably reduce the processing of redundant information.

The hit-or-miss transformation used for shape matching needs complete knowledge of the shapes within image I. Typically, the shape recognition operation can be successful only if the object shape in image $I$ is perfect. This condition seems impractical because normally the object shape operating in the acquired digital image cannot match exactly the shape of that used to construct the SE. In Scction 3, we present a hit-or-miss transformation whose SEs do not need to exactly match the object shape in image $I$ for successful operation. Most often acquired shapes are indeterminate. The hit-or-miss transformation will need to utilize a pair of SEs which satisfy certain conditions while the operations for matching the indeterminate shapes have remained unchanged. In Section 4, we prove that the window $W$ in the hit-or-miss transformation is not necessarily a part of the SEs if the SEs are the boundaries of object shapes. This is also true for matching indeterminate shapes resulting from acquiring digital images in noisy environments.

\section{HIT-OR-MISS TRANSFORMATION FOR SHAPE MATCHING}

The hit-or-miss operation employing the shape to be recognized as an SE contains a degree of redundancy. The redundancy is in the form of unnecessary computation during erosion operations. An automated shape recognition process consists of two erosions with the structuring elements $A$ and ( $W \cap A^{\mathrm{c}}$ ), respectively. Let $I$ be a given 2-D image and $A$ be some specific shape in $I$ to be recognized. The erosion of $I$ by $A$ can be written as the set

$$
I \ominus A=\bigcap_{a \in A} I-a,
$$

and likewise the erosion of $I^{\mathrm{c}}$ (complement of $I$ ) by ( $W \cap$ $\left.A^{c}\right)$ is the set

$$
I^{\mathrm{c}} \ominus\left(W \cap A^{\mathrm{c}}\right)=\bigcap_{a \in\left(W \cap A^{\mathrm{c}}\right)} I^{\mathrm{c}}-a .
$$

As noted, the window $W$ is chosen so that the object $A$ is a subset of $W$. The erosion involves shifting an image frame in directions according to the points in $A$ and then intersecting the shifted frames. The number of pixels within the desired window $W$ determines the number of times the image is to be shifted and the number of intersections to be completed. This has been proven to be a computationally intensive process. Do we really need all points within set $A$ or set $\left(W \cap A^{c}\right)$ in order to accomplish automated shape recognition using a hit-or-miss operator? In other words, given a random shape $A$, can we find an essential subset of $A$ with which to perform the matching process with the same effect as if $A$ were used? The answer to this question is that such essential subsets do exist. In the following, a new and more efficient approach is described. The definition of shape recognition used here is that the recognition operation is accomplished if the locations of the shape are identified within the image.

Let $\partial A=A \backslash(A \ominus \Delta)$ stand for the boundary of shape $A$ where the notation $\backslash$ is for set difference and $\Delta$ is a set including the origin $(0,0)$ and its immediate neighboring points. We define a shape $A$ in image $I$ separated from others by window $W$ as that for a given $p, W_{p} \cap \Lambda_{p}=A_{p}$ and $W_{p} \cap B_{q}=\emptyset$ for any shape $B$ in image $I$ at the location $q \neq p$. The following theorem presents an efficient solution to the automated shape recognition problem using hit-or-miss transformations.

THEOREM 1. If shape $A, A \subset W$, in image I is separated from other shapes by $W$, and either $\partial A \subseteq B$ or $\partial A^{c} \subseteq B^{c}$, then the shape $A$ occurs in the image I at, and only at, the locations represented by set $L$,

$$
L=[I \ominus \partial A] \cap\left[I^{\mathfrak{c}} \ominus \partial\left(W \cap A^{\mathrm{c}}\right)\right] .
$$

This theorem states that the subset found to replace the shape is in effect the boundary of the object, and therefore, erosion is reduced to using only those points on the boundary of the object shape. The computational procedure is to first find the boundary of the object. Since the object, or the shape, to be recognized is known a priori, boundary information can be easily recalled from a database. Note that for a group of known shapes, it is possible to find an optimal subset which may be used for such recognition problems. However, the approach provided here assumes no knowledge of other shapes which may appear in the acquired image. To prove the theorem, the following lemmas are useful.

LEMma 1. Let window $W$ be a binary set such that $W$ $\cap A=A$ and $W \cap B=B$, and if $A \neq B$, and either $\partial B \nsubseteq A$ or $\partial B^{c} \nsubseteq A^{c}$, then

$$
\lceil A \ominus \partial B\rceil \cap\left[A^{\mathrm{c}} \ominus \partial\left(W \cap B^{\mathrm{c}}\right)\right]=\emptyset .
$$


Proof. There are three possible relationships between $A$ and $B$. (1) There exists a $p$ such that $A_{p} \subset B$; (2) there exists a $p$ such that $A_{p} \supset B$; and (3) there does not exist a $p$ such that $A_{p} \subset B$ or $A_{p} \supset B$, but there exists an $x$ such that $A_{x} \cap B \neq \emptyset$.

In (1), $(A \ominus B)_{p}=\left(A_{p} \ominus B\right)$. Since $A_{p} \subset B$ and $A_{p} \ominus$ $\partial B=\bigcap_{b \in \partial B}\left(A_{p}\right)_{-b}$, then there exists at least one $b$ such that $b \in \partial B$ but $b \notin A_{p}$. For every $a \in A_{p}, \cap_{b \in B}\{a-b\}=$ $\emptyset$. Hence $\cap_{b \in \partial B}\left(A_{p}\right)_{-b}=\emptyset$.

In (2), using $A \ominus B=\left(A^{\mathrm{c}} \oplus B\right)^{\mathrm{c}}$ then $(A \ominus \partial B) \cap\left(A^{\mathrm{c}} \ominus\right.$ $\left.\partial\left(W \cap B^{\mathrm{c}}\right)\right)=\left[\left(A_{p} \ominus \partial B\right) \cap\left(A_{p} \oplus \partial\left(W \cap B^{\mathrm{c}}\right)\right)^{\mathrm{c}}\right]_{-p}$. That is, there is a set $\{z\}$ such that $z \in A_{p} \ominus \partial B$ and $z \notin A_{p} \oplus$ $\partial\left(W \cap B^{c}\right)$. But $A_{p} \ominus \partial B \subseteq\left(A_{p}\right)_{-b \forall b \in \partial B}$ and there exists at least one $b$ such that $\left(A_{p}\right)_{-b} \subseteq A_{p} \oplus \partial\left(W \cap B^{c}\right)$. That is, $A_{p} \ominus \partial B \subseteq A_{p} \oplus \partial\left(W \cap B^{c}\right)$. This is a contradiction to $z \in$ $A_{p} \ominus \partial B$ and $z \notin A_{p} \oplus \partial\left(W \cap B^{\mathfrak{c}}\right)$.

In (3), because for any $p, A_{p} \not \subset B$ or $A_{p} \not \supset B$ for any $p$. Hence, there exists at least one $b \in \partial B$ such that $b \notin A_{p}$. That is, $\{z: z+\partial B \subseteq A\}=\emptyset$.

Q.E.D.

LEMMA 2. Let $A \subset Z^{2}$, and $\partial A$ be the boundary of $A$; then

$$
A \ominus A=A \ominus \partial A=\{(0,0)\} .
$$

Proof. (1) $A \ominus A=\{(0,0)\}$. By definition, for $p \in A \ominus$ $A, p+A \subseteq A$ iff $p=(0,0)$.

(2) $A \ominus \partial A=\{(0,0)\}$. Since $\partial A \subseteq A, A \ominus \partial A \supseteq A \ominus A=$ $\{(0,0)\}$. It is necessary to prove $A \ominus \partial A \subseteq\{(0,0)\}$. Assume that $q \in A \ominus \partial A$, then $q+\partial A \subseteq A$. There is at least one $z \in \partial A$ such that if $q \neq(0,0)$, then $q+z \notin A$ since $z$ is on the boundary of $A$. Therefore, $q=(0,0)$.

Q.E.D.

Because $A_{p} \ominus B=(A \ominus B)_{p}, A \ominus \partial A=\{(0,0)\}$, and $A^{\mathrm{c}} \ominus$ $\partial\left(W \cap A^{\mathrm{c}}\right) \supseteq\left(W \cap A^{\mathrm{c}}\right) \ominus \partial\left(W \cap A^{\mathrm{c}}\right)=\{(0,0)\}$, we can conclude from Lemmas 1 and 2 that

$$
\left[A_{p} \ominus \partial A\right] \cap\left[A_{p}^{\mathrm{c}} \ominus \partial\left(W \cap A^{\mathrm{c}}\right)\right]=\{p\} .
$$

We define $A$ and $B$ as being separated if any immediate neighboring point of $A$ does not belong to $B$; that is, for any $z \in \partial A^{\mathrm{c}}, z \notin B$.

Proposition 1. If $A$ and $B$ are separated, then

$$
(A \cup B) \ominus K=(A \ominus K) \cup(B \ominus K) .
$$

Proof. It is known that in general $[2,12]$

$$
(A \cup B) \ominus K \supseteq(A \ominus K) \cup(B \ominus K) .
$$

For $p \in(A \cup B) \ominus K, p+K \subseteq(A \cup B)$. Since $A$ and $B$ are separated, either $p+K \subseteq A$ or $p+K \subseteq B$, that is $p \in$ $A \ominus K$ or $p \in B \ominus K$, which means that

$$
(A \cup B) \ominus K \subseteq(A \ominus K) \cup(B \ominus K) .
$$

Combining Eq. (9) with Eq. (8) leads to Eq. (7).

Q.E.D.

Proof of Theorem 1. Because the shapes in image $I$ are separated by window $W$, we have

$$
I=\left(\bigcup_{p \in P} A_{p}\right) \cup\left(\bigcup_{q \in Q} B_{q}\right),
$$

where $B$ represents any shape other than $A$. Then, the erosion of $I$ by $\partial A$ becomes

$$
I \ominus \partial A=\left(\bigcup_{p \in P} A_{p} \ominus \partial A\right) \cup\left(\bigcup_{q \in Q} B_{q} \ominus \partial A\right) .
$$

By the duality property that $A \ominus B=\left(A^{\mathrm{c}} \oplus B\right)^{\mathrm{c}}$, then the erosion of $I^{\mathrm{c}}$ by $\partial\left(W \cap A^{\mathrm{c}}\right)$ becomes

$$
\begin{aligned}
& I^{\mathrm{c}} \ominus \partial\left(W \cap A^{\mathrm{c}}\right) \\
= & {\left[\left(\bigcup_{p \in P} A_{p}\right) \cup\left(\bigcup_{q \in Q} B_{q}\right)\right]^{\mathrm{c}} \ominus \partial\left(W \cap A^{\mathrm{c}}\right) } \\
= & \left\{\left[\left(\bigcup_{p \in P} A_{p}\right) \cup\left(\bigcup_{q \in Q} B_{q}\right)\right] \oplus \partial\left(W \cap A^{\mathrm{c}}\right)\right\}^{\mathrm{c}} \\
= & \left\{\left[\bigcup_{p \in P} A_{p} \oplus \partial\left(W \cap A^{\mathrm{c}}\right)\right] \cup\left[\bigcup_{q \in Q} B_{q} \oplus \partial\left(W \cap A^{\mathrm{c}}\right)\right]\right\}^{\mathrm{c}} \\
= & {\left[\bigcap_{p \in P}\left(A_{p} \oplus \partial\left(W \cap A^{\mathrm{c}}\right)\right)^{\mathrm{c}}\right] \cap\left[\bigcap_{q \in Q}\left(B_{q} \oplus \partial\left(W \cap A^{\mathrm{c}}\right)\right)^{\mathrm{c}}\right] } \\
= & {\left[\bigcap_{p \in P}\left(A_{p}^{\mathrm{c}} \ominus \partial\left(W \cap A^{\mathrm{c}}\right)\right)\right] \cap\left[\bigcap_{q \in Q}\left(B_{q}^{\mathrm{c}} \ominus \partial\left(W \cap A^{\mathrm{c}}\right)\right)\right] . }
\end{aligned}
$$

Therefore, the set $L$ becomes

$$
\begin{aligned}
L= & \left\{\left(\bigcup_{p \in P} A_{p} \ominus \partial A\right) \cup\left(\bigcup_{q \in Q} B_{q} \ominus \partial A\right)\right\} \\
& \cap\left\{\left[\bigcap_{p \in P}\left(A_{p}^{\mathrm{c}} \ominus \partial\left(W \cap A^{\mathrm{c}}\right)\right)^{\mathrm{c}}\right]\right. \\
& \left.\cap\left[\bigcup_{q \in Q}\left(B_{q}^{\mathrm{c}} \ominus \partial\left(W \cap A^{\mathrm{c}}\right)\right)^{\mathrm{c}}\right]\right\} \\
= & \left\{\bigcup _ { p \in P } \left[\left(A_{p} \ominus \partial A\right) \cap\left(A_{p}^{\mathrm{c}} \ominus \partial\left(W \cap A^{\mathrm{c}}\right)\right)\right.\right. \\
& \bigcap_{k \in P, k \neq p}\left(A_{k}^{\mathrm{c}} \ominus \partial\left(W \cap A^{\mathrm{c}}\right)\right) \\
& \left.\left.\bigcap_{q \in Q}\left(B_{q}^{\mathrm{c}} \ominus \partial\left(W \cap A^{\mathrm{c}}\right)\right)\right]\right\} \\
& \cup\left\{\bigcup _ { q \in Q } \left[\left(B_{q} \ominus \partial A\right) \bigcap\left(B_{k}^{\mathrm{c}} \ominus \partial\left(W \cap A^{\mathrm{c}}\right)\right)\right.\right. \\
& \left.\left.\bigcap_{p \in P}\left(A_{p}^{\mathrm{c}} \ominus \partial\left(W \cap A^{\mathrm{c}}\right)\right)\right]\right\} .
\end{aligned}
$$


Because all shapes in $I$ are separated by window $W, p \notin$ $A_{k} \oplus \partial\left(W \cap A^{\mathrm{c}}\right)$ for $p \in P$ and $p \neq k$, and $p \notin B_{q} \oplus \partial(W \cap$ $\left.A^{\mathrm{c}}\right)$ for $p \in P$ and $q \in Q$. Thus, $p \in\left(A_{k} \oplus \partial\left(W \cap A^{\mathrm{c}}\right)\right)^{\mathrm{c}}=$ $\left(A_{k}^{\mathrm{c}} \ominus \partial\left(W \cap A^{\mathrm{c}}\right)\right)$ and $p \in\left[B_{q} \oplus \partial\left(W \cap A^{\mathrm{c}}\right)\right]^{\mathrm{c}}=B_{q}^{\mathrm{c}} \ominus$ $\partial\left(W \cap A^{\mathrm{c}}\right)$. Therefore,

$$
\begin{aligned}
& \bigcup_{p \in P}\left[\left(A_{p} \ominus \partial A\right) \cap\left(A_{p}^{\mathrm{c}} \ominus \partial\left(W \cap A^{\mathrm{c}}\right)\right)\right. \\
& \left.\bigcap_{k \in P, \neq p}\left(A_{k}^{\mathrm{c}} \ominus \partial\left(W \cap A^{\mathrm{c}}\right)\right) \bigcap_{q \in Q}\left(B_{q}^{\mathrm{c}} \ominus \partial\left(W \cap A^{\mathrm{c}}\right)\right)\right] \\
= & \{p \in P\}
\end{aligned}
$$

and

$$
\begin{aligned}
& \bigcup_{q \in Q}\left[B_{q} \ominus \partial A \bigcap_{k \in Q} B_{q}^{\mathrm{c}} \ominus \partial\left(W \cap A^{\mathrm{c}}\right)\right. \\
& \left.\bigcap_{p \in P}\left(A_{p}^{\mathrm{c}} \ominus \partial\left(W \cap A^{\mathrm{c}}\right)\right)\right] \\
= & \bigcup_{q \in Q}\left[B_{q} \ominus \partial A\right) \cap\left(B_{q}^{\mathrm{c}} \ominus \partial\left(W \cap A^{\mathrm{c}}\right)\right) \\
& \left.\bigcap_{k \in K, k \neq q}\left(B_{k}^{\mathrm{c}} \ominus \partial\left(W \cap A^{\mathrm{c}}\right)\right) \bigcap_{p \in P}\left(A_{p}^{\mathrm{c}} \ominus \partial\left(W \cap A^{\mathrm{c}}\right)\right)\right] \\
= & \emptyset .
\end{aligned}
$$

This concludes that for each $p \in P$, it corresponds to $A_{p}$, a shifted shape $A$, in $I$.

Q.E.D.

Theorem 1 establishes that the structuring elements are subsets of the shapes $A$ and $\left(W \cap A^{\mathrm{c}}\right)$ and that these subsets are actually the boundaries of $A$ and $\left(W \cap A^{\mathrm{c}}\right.$ ). Therefore, the computation time required for the recognition process is governed by the number of points on the boundaries of $A$ and ( $W \cap A^{c}$ ), which corresponds to the number of shifts and intersections required. We consider a simple example which will provide an intuitive appreciation for the theorem presented here. Suppose that the shape $A$ is a square of size $16 \times 16$ pixels and the size of window $W$ is $32 \times 32$. Then eroding image $I$ by structuring element $A$ requires that the image $I$ be shifted 256 times and the resulting shifted images be intersected, respectively. Eroding the complement of image, $I^{\mathrm{c}}$, by the structuring element $\left(W \cap A^{\mathrm{c}}\right)$ requires a total of 768 shifts and intersections. The total number of shifts (with intersections) is determined by the window $W$ (which is in turn determined by the size of $A$ ) and is found in this example to be 1022 . When using Theorem 1 , the boundary of $A, \partial A$, is employed as a structuring element. There are only 62 points within the set $\partial A$ and 192 points within the set $\partial\left(W \cap A^{\mathrm{c}}\right)$. Therefore, the total number of pairs of shifts and intersections for this example is 254 , which is about one-quarter of the total number of operations required when using $\left\{A, W \cap A^{c}\right\}$ as the SEs. The impact of the theorems presented here is the resulting efficiency in the computational requirement.

Figure 1 shows an example of discriminating one object from among others. Figure la displays shape $A$ to be recognized. Figure $1 \mathrm{~b}$ is the image which contains 16 different shapes including $A$. Figure $1 c$ shows the erosion of $I$ by $\partial A$, while Fig. $1 \mathrm{~d}$ displays the result of $I^{\mathfrak{c}} \ominus \partial(W \cap$ $A^{\mathrm{c}}$ ). Shape recognition (by the method of locating the object) is completed by the final step of intersection of the set in Fig. 1c with the set in Fig. 1d. The result is shown in Fig. 1e.

For the purpose of inspection, or other related feature detection requirements in many practical situations, the acquired image will contain the shape which may not be exactly true to form. That is, imperfections in the image acquisition process will cause the image data to contain shape distortions compared to those in the database from which the structuring elements are extracted. Let $\Gamma$ stand for the set of all possible variation parameters. Then the imperfect shape appearing at $z$ in $I$ is represented by $A_{z}(\gamma), \gamma \in \Gamma$

Similar to Theorem 1, the hit-or-miss process can be accomplished as described according the corollary below.

COROLlary 1. The pattern $\{A(\gamma)\}$ occurs in the image $I$ at the locations represented by set $L$,

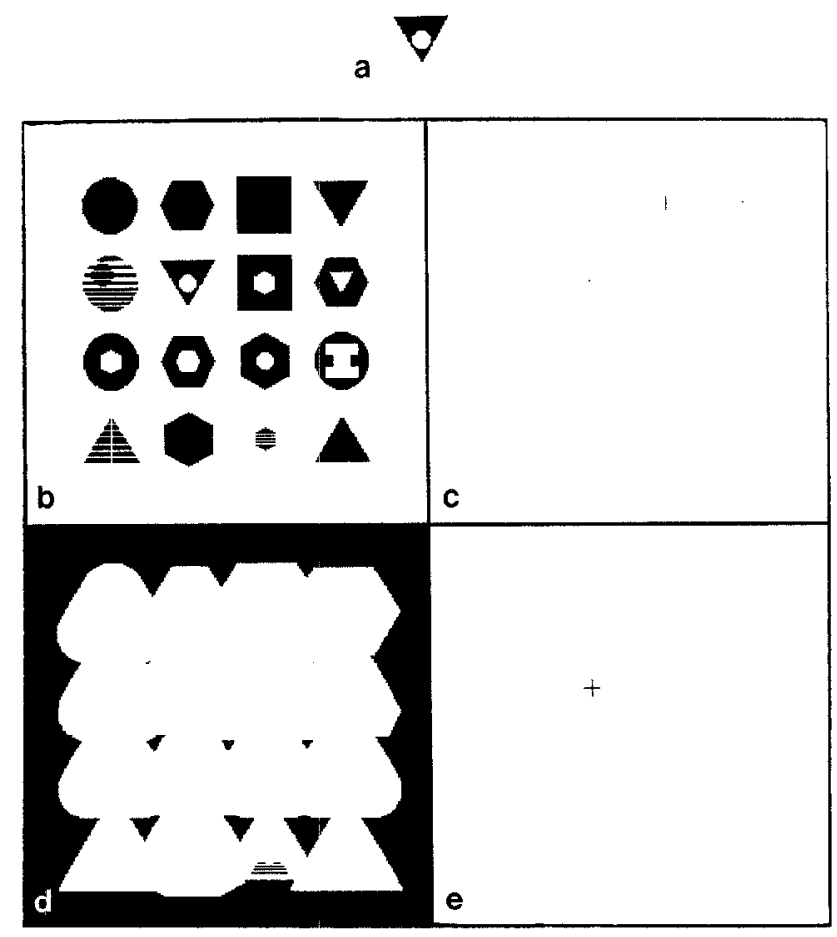

FIG. 1. Shape recognition by location of a specified object. (a) Shape $A$ to be recognized; (b) image $I$ with 16 shapes; (c) $I \ominus A$; (d) $I^{\circ} \ominus$ $\partial\left(W \cap A^{\mathrm{c}}\right) ;(\mathrm{e})[I \ominus \partial A] \cap\left[I^{\mathrm{c}} \bigcirc \partial\left(W \cap A^{\mathrm{c}}\right)\right]$. 


$$
L=\bigcup_{\gamma \in \Gamma}[I \ominus \partial A(\gamma)] \cap\left[I^{\mathrm{c}} \ominus \partial\left(W \cap A^{\mathrm{c}}(\gamma)\right)\right]
$$

Corollary 1 states that for the imperfect shapes derived from an ideal shape $A$, the recognition process can be realized by simply utilizing exhaustively all possible variations of that shape. This approach would be able to provide not only the object location in the image but also the information regarding the shape imperfection. That is, by trying a hit-or-miss operator on all possible variations of the shape, $\{A(\gamma)\}$, an accurate location of the shape can be detected. However, the process requires a prohibitively large amount of computer time to complete. It would be impractical to exhaustively scarch over all possibilities even in the simple case of a minor deterioration of the shape boundary.

To avoid the immense computational requirement for hit-or-miss operations in the case of imperfect shapes, we find that the computational intensity can be reduced if the actual shape variation is of no concern to us. What we need to know is only the locations of the target shapes without regard to their possible variations. 'Therefore, a shape will be detected on the basis of its original geometric structure and not on the basis of its exact imperfection.

\section{RECOGNITION OF IMPERFECT SHAPES FROM INDETERMINATE VARIATIONS OF PERFECT SHAPES}

As introduced in Sections 1 and 2, the shape recognition process is achieved assuming that the shapes within an image are known exactly. For the case of imperfect shapes, we need to find a process which should be able to recognize the target shape in a noisy image without making the recognition process unduly complicated.

The imperfect shapes can be viewed as perfect shapes that have had noise added. Noise is unavoidable during the process of image acquisition and digitization. Salt and pepper noise is typically seen in an image acquired without averaging over a number of consecutive frames. This type of noise is normally small in size and can be easily removed by "opening-closing" morphological filtering operations on either the gray-scale image or its binary version. Careful choice of a structuring element for the opening-closing operations can eliminate salt and pepper noise without affecting the geometric representation of shapes in the image.

A major source of shape distortion is the nonlinear thresholding operation which converts a gray-scale image into a binary image. In many situations, an object is not appropriately represented in the image, e.g., object motion, focus blurring, improper camera, and/or light settings. After all, the shape cannot be made exactly the same as in sample space. Hence, the recognition of per- fect shape is only an ideal situation. After a thresholding decision is made upon the image, the resulting binary version is the input to the hit-or-miss transformation. Shape imperfections can be treated as a perfect shape affected by noise. The random variations along the boundaries of a shape are most frequently seen. We consider these random variations as indeterminate shapes.

To begin the analysis of recognizing imperfect shapes, we first establish some necessary terminology. We define the sample space, $\mathbf{A}$, as a set of shapes possibly appearing in images upon which the recognition process is to be performed. Any element in the sample space is thought of as a perfect shape. We define the relevant sample space, $\overline{\mathbf{A}}$, as a collection of all possible variations of the shapes in the sample space. That is, $\tilde{\mathbf{A}}$ contains a set of indeterminate shapes which are variations of $\mathbf{A}$. Assume that the shape $A$ is to be recognized. We need to find an SE which best describes the property of shape $A$ and at the same time overcome the effects of indeterminate variations of $A$. Since there are two steps in the hit-or-miss opcration, the SE used for eroding the foreground of the binary image must be able to preserve information concerning the shape $\tilde{A}$. In addition, the SE used for eroding the background of the binary image must preserve information relating to the windowed complement of $\tilde{A}$. Both SEs should also be as geometrically different as possible from the other shapes in $\tilde{\mathbf{A}}$ which are not target objects. The following theorem states that when certain conditions regarding SEs are satisfied, the desired object shape can be recognized.

THEOREM 2. Let $A_{i}$ be a shape in the sample space $\boldsymbol{A}$, $\tilde{A}_{i}$ be an indeterminate variation of $A_{i}$ in image $I$ where $\left\{\tilde{A}_{i}, I\right\} \in Z^{2}$, and $W$ be a window such that $A_{i} \subset W$ and $\tilde{A}_{i} \subset W$. Let $A_{i}$ be the lower bound of variations of shape $A_{i}$ and $\bar{\Lambda}_{i}$ be the upper bound of variations of shape $\Lambda_{i}$ and $A_{i} \subseteq \overline{A_{i}} \subset W$, such that the following conditions are satisfied:

(1) $\underline{A_{i}} \subseteq \tilde{A}_{i}$ and $W \cap \bar{A}_{i}^{\mathrm{c}} \subseteq W \cap \tilde{A}_{i}^{\mathrm{c}}$,

(2) for any $x, \underline{A_{i}}+x \nsubseteq \tilde{A}_{j}$ or $W \cap\left(\bar{A}_{i}^{c^{\prime}}+x\right) \nsubseteq W \cap \tilde{A}_{j}^{c}$ for $j \neq i$.

Let $E=\frac{1}{2}\left(\overline{A_{i}} \ominus A_{i}\right)$. If $\tilde{A}_{i}$ is separated from other shapes in I by $W$, then $\tilde{A}_{i}$, hence $A_{i}$, is at location $p$ in $I$, where

$$
p \in\left[I \ominus \underline{A_{i}}\right] \cap\left[I^{\mathrm{c}} \ominus\left(W \cap \bar{A}_{i}^{\mathrm{c}}\right)\right] \subseteq \bigcup_{q \in Q} E_{q}
$$

and $Q=\left\{z: z \in\left[I \ominus A_{i} \cap I^{\mathrm{c}} \ominus\left(W \cap A_{i}^{\mathrm{c}}\right)\right] /\right.$ as if $A_{i}$ in $I$ were perfect $\}$.

Proof. Before proceeding with the image $I$, the following development will assist the proof. From condition (1), $\{(0,0)\}=\tilde{A}_{i} \ominus \tilde{A}_{i} \subseteq \tilde{A}_{i} \ominus \underline{A_{i}}$ and $\{(0,0)\}=\tilde{A}_{i}^{\mathrm{c}} \ominus(W \cap$ $\left.\tilde{A}_{i}^{\mathrm{c}}\right) \subseteq \tilde{A}_{i}^{\mathrm{c}} \ominus\left(W \cap \bar{A}_{i}^{\mathrm{c}}\right)$. Hence,

$$
\left[\tilde{A}_{i} \ominus \underline{A_{i}}\right] \cap\left[\tilde{A}_{i}^{\mathrm{c}} \ominus\left(W \cap \bar{A}_{i}^{\mathrm{c}}\right)\right] \supseteq\{(0,0)\} .
$$


From condition (2), for $A_{j} \neq A_{i}, \tilde{A}_{i}+x \nsubseteq \tilde{A}_{j}$ or $W \cap$ $\left(\tilde{A}_{i}^{\mathrm{c}}+x\right) \nsubseteq\left(W \cap \tilde{A}_{j}^{\mathrm{c}}\right)$. Then,

$$
\left[\tilde{A}_{j} \ominus \underline{A_{i}}\right] \cap\left[\tilde{A}_{j}^{\mathrm{c}} \ominus\left(W \cap \bar{A}_{i}^{\mathrm{c}}\right)\right]=\emptyset .
$$

To determine the size of cluster $C=\left[\tilde{A}_{i} \ominus \underline{A}_{i}\right] \cap\left[\tilde{A}_{i}^{\mathrm{c}} \ominus\right.$ $\left.\left(W \cap \bar{A}_{i}^{\mathrm{c}}\right)\right]$, let $\vec{v}$ be a direction along which all points in $C$ are examined. Let $V_{1}$ be a set of points along $\vec{v}$ such that $\underline{A_{i}}+v_{1} \subseteq \tilde{A}_{i}$, where $v_{1} \in V_{1}$. Let $V_{2}$ be a set of points along $-\vec{v}$ such that $W \cap \bar{A}_{i}^{\mathrm{c}}+v_{2} \subseteq \tilde{A}_{i}^{\mathrm{c}}$, where $v_{2} \in V_{2}$. Let $V_{3}$ be a set of points along $-\vec{v}$ such that $\underline{A_{i}}+v_{3} \subseteq \tilde{A}_{i}$, where $v_{3} \in V_{3}$, and let $V_{4}$ be a set of points along $\vec{v}$ such that $W \cap \bar{A}_{i}^{\mathrm{c}}+v_{4} \subseteq \tilde{A}_{i}^{\mathrm{c}}$, where $v_{4} \in V_{4}$. Then, the subset of the resultant hit-or-miss transformation along the directions of $\vec{v}$ and $-\vec{v}$ is the set described as

$$
\begin{aligned}
& {\left[\left(\tilde{A}_{i} \ominus{\underline{A_{i}}}\right) \cap\left(\tilde{A}_{i}^{\mathrm{c}} \ominus W \cap \bar{A}_{i}^{\mathrm{c}}\right)\right]_{\text {along } \tilde{v} \text { and }-\vec{v}} } \\
= & {\left[V_{1} \cup V_{3}\right] \cap\left[V_{2} \cup V_{4}\right] } \\
= & \left(V_{1} \cap V_{4}\right) \cup\left(V_{2} \cap V_{3}\right)
\end{aligned}
$$

because $V_{1} \cap V_{2}=\emptyset$ and $V_{3} \cap V_{4}=\emptyset$. For $\left(V_{1} \cap V_{4}\right) \cup$ $\left(V_{2} \cap V_{3}\right)$ to be a maximal set, let $\overline{V_{1}}=\overline{V_{4}}=\frac{1}{2}\left\{x: x+\underline{A_{i}} \subseteq\right.$ $\overline{A_{i}} / x$ on $\left.\vec{v}\right\}=\frac{1}{2}\left\{x: x+W \cap \bar{A}_{i}^{\mathrm{c}} \subseteq A_{i}^{\mathrm{c}} / x\right.$ on $\left.\vec{v}\right\}$ and $\overline{V_{2}}=\overline{V_{3}}=$ $\frac{1}{2}\left\{x: x+A_{i} \subseteq \overline{A_{i}} / x\right.$ on $\left.-\vec{v}\right\}=\frac{1}{2}\left\{\overline{x: x}+W \cap \overline{A_{i}^{\mathrm{c}}} \subseteq \underline{A_{i}^{\mathrm{c}}} / x\right.$ on $-\vec{v}\}$. Therefore, $\left(V_{1} \cap V_{4}\right) \cup\left(V_{2} \cap V_{3}\right) \subseteq \frac{1}{2}\left\{x: x+\bar{A}_{i} \subseteq\right.$

$\left.\overline{A_{i}}\right\}_{\text {on } v}$ and $-\vec{v}$. We conclude that the resultant set from the hit-or-miss transformation is the union of the subsets along all possible directions. That is,

$$
\begin{aligned}
& \left(\tilde{A}_{i} \ominus \underline{A_{i}}\right) \cap\left(\tilde{A}_{i}^{\mathrm{c}} \ominus W \cap \overline{A_{i}^{\mathrm{c}}}\right) \\
\subseteq & \bigcup_{\text {all } \vec{v} \text { and }-\vec{v}} \frac{1}{2}\left\{p: p \in\left(\overline{A_{i}} \ominus \underline{A_{i}}\right) / p \text { is along } \vec{v} \text { or }-\vec{v}\right\} \\
= & \frac{1}{2}\left(\overline{A_{i}} \ominus \underline{A_{i}}\right) \\
= & E .
\end{aligned}
$$

Now proceed with the hit-or-miss transformation on image $I$ using $A_{i}$ and $\left(W \cap \bar{A}_{i}^{\mathrm{c}}\right)$ as SEs. For any shape $S_{k}$ $\in \mathbf{A}$, we have

$$
\begin{aligned}
& {\left[I \ominus \underline{A_{i}}\right] \cap\left[I^{\mathrm{c}} \ominus\left(W \cap \bar{A}_{i}^{\mathrm{c}}\right)\right] } \\
= & {\left[I \ominus \underline{A_{i}}\right] \cap\left[I \oplus\left(W \cap \bar{A}_{i}^{\mathrm{c}}\right)\right]^{c} } \\
= & {\left[\left(\bigcup_{k} \tilde{S}(k)_{p_{k}}\right) \ominus \underline{A_{i}}\right] \cap\left[\left(\bigcup_{k} \tilde{S}(k)_{p_{k}}\right) \oplus\left(W \cap \bar{A}_{i}^{\mathrm{c}}\right)\right]^{\mathrm{c}} } \\
= & {\left[\bigcup_{k} \tilde{S}(k)_{p_{k}} \ominus A_{i}\right] \cap\left[\bigcup_{k} \tilde{S}(k)_{p_{k}} \oplus\left(W \cap \bar{A}_{i}^{\mathrm{c}}\right)\right]^{\mathrm{c}}(30) }
\end{aligned}
$$

$$
\begin{aligned}
& =\left[\bigcup_{k}\left(\tilde{S}(k)_{p_{k}} \ominus \underline{A_{i}}\right)\right] \cap\left[\bigcup_{k}\left(\tilde{S}(k)_{p_{k}} \oplus\left(W \cap \bar{A}_{i}^{\mathrm{c}}\right)\right)^{\mathrm{c}}\right] \\
& =\left\lfloor\bigcup_{k}\left(\tilde{S}(k)_{p_{k}} \ominus \underline{A_{i}}\right)\right] \cap\left[\bigcup_{k}\left(\tilde{S}(k)_{p_{k}}^{\mathrm{c}} \ominus\left(W \cap \bar{A}_{i}^{\mathrm{c}}\right)\right)\right] \\
& =\bigcup_{k}\left[\left(\tilde{S}(k)_{p_{k}} \ominus \underline{A_{i}}\right) \bigcap_{l}\left(\tilde{S}(l)_{p_{i}}^{\mathrm{c}} \ominus\left(W \cap \bar{A}_{i}^{\mathrm{c}}\right)\right)\right] \\
& =\left[\bigcup_{k}\left(\tilde{S}(k)_{p_{k}} \ominus \underline{A_{i}}\right) \cap\left(\tilde{S}(k)_{p_{k}}^{\mathrm{c}} \ominus\left(W \cap \bar{A}_{i}^{\mathrm{c}}\right)\right)\right] \\
& \cap\left[\bigcap_{p_{l} \neq p_{k}} \tilde{S}(l)_{p_{l}}^{\mathfrak{c}} \ominus\left(W \cap \bar{A}_{i}^{\mathrm{c}}\right)\right] \\
& =\bigcup_{k}\left[\left(\bar{S}(k)_{p_{k}} \ominus \underline{A_{i}}\right) \cap\left(\bar{S}(k)_{p_{k}}^{\mathrm{c}} \ominus\left(W \cap \bar{A}_{i}^{\mathrm{c}}\right)\right)\right] \\
& =\bigcup_{k^{\prime}}\left[\left(\tilde{A}_{i_{p_{k^{\prime}}}} \ominus \underline{A_{i}}\right) \cap\left(\tilde{A}_{i_{p_{k^{\prime}}}}^{\mathrm{c}} \ominus\left(W \cap \bar{A}_{i}^{\mathrm{c}}\right)\right)\right] \\
& \bigcup_{\tilde{S}(k) \neq \bar{A}_{i}}\left[\tilde{S}(k)_{p_{k}} \ominus A_{i} \cap \tilde{S}(k)_{\mathrm{p}_{k}}^{\mathrm{c}} \ominus\left(W \cap \bar{A}_{i}^{\mathrm{c}}\right)\right] \\
& =\bigcup_{k^{\prime}}\left[\left(\tilde{A}_{i_{p_{k^{\prime}}}} \ominus \underline{A_{i}}\right) \cap\left(\tilde{A}_{i_{p_{k^{\prime}}}}^{\mathrm{c}} \ominus\left(W \cap \bar{A}_{i}^{\mathrm{c}}\right)\right)\right] \\
& =\bigcup_{k^{\prime}}\left[\left(\tilde{A}_{i} \ominus \underline{A_{i}}\right)_{p_{k^{\prime}}} \cap\left(\tilde{A}_{i}^{\mathrm{c}} \ominus\left(W \cap \overline{A_{i}^{\mathrm{c}}}\right)\right)_{p_{k^{\prime}}}\right] \\
& =\bigcup_{k^{\prime}}\left[\left(\tilde{A}_{i} \ominus \underline{A_{i}}\right) \cap\left(\tilde{A}_{i}^{\mathrm{c}} \ominus\left(W \cap \bar{A}_{i}^{\mathrm{c}}\right)\right)_{p_{k^{\prime}}}\right. \\
& \subseteq \bigcup_{k^{\prime}} E_{p^{\prime}} .
\end{aligned}
$$

The steps arising from Eq. (32) to Eq. (33) are due to the fact that the $\tilde{S}(k)_{p k}$ 's are separated by $W$. Moving from Eq. (34) to Eq. (35) is possible because the $\tilde{S}(k)_{p_{k}}$ 's are also separated by $W$, which concludes $x \in \tilde{S}(l)_{\mathrm{p}_{1}}^{\mathrm{c}} \ominus(W \cap$ $\left.\bar{A}_{i}^{\mathrm{c}}\right)$. This leads to either $x \in \tilde{S}(k)_{p_{k}} \ominus \underline{A_{i}}$ or $x \in \tilde{S}(k)_{p_{k}}^{\mathrm{c}} \ominus$ $\left(W \cap \bar{A}_{i}^{\mathrm{c}}\right)$.

Q.E.D.

On the basis of the discussion concerning use of the shape boundaries as SEs, we now formulate a theorem for the case of indeterminate variations in the shapes.

THEOREM 3. Let $A_{i}$ be a shape in the sample space $\mathbf{A}$, $\tilde{A}_{i}$ be an indeterminate variation of $A_{i}$ in image I where $\left\{A_{i}, I\right\} \in Z^{2}$, and $W$ be a window such that $A_{i} \subset W$ and $\tilde{A}_{i} \subset W$. Let $A_{i}$ be the lower bound of variations of shape $A_{i}$ within $W \overline{\text { and }} \overline{A_{i}}$ be the upper bound of variations of shape $A_{i}$ and $\underline{A}_{i} \subset \overline{A_{i}} \subset W$, such that the following conditions are satisfied:

(1) $\partial \underline{A_{i}} \subseteq \tilde{A}_{i}$ and $\partial\left(W \cap \bar{A}_{i}^{\mathrm{c}}\right) \subseteq\left(W \cap \tilde{A}_{i}^{\mathrm{c}}\right)$,

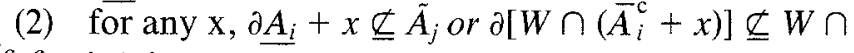
$\tilde{A}_{j}^{\mathrm{c}}$ for $j \neq i$. 
Let $E=\frac{1}{2}\left(\underline{A}_{i} \ominus \underline{A_{i}}\right)$. If $\tilde{A}_{i}$ is separated from others in I by $W$, then $\tilde{A}_{i}$, hence $A_{i}$, is at location $p$, where

$$
p \in\left[I \ominus \partial A_{i}\right] \cap\left[I^{\mathrm{c}} \ominus \partial\left(W \cap \bar{A}_{i}^{\mathrm{c}}\right)\right] \subseteq \bigcup_{q \in Q} E_{q}
$$

and $Q=\left\{z: z \in\left[I \ominus A_{i} \cap I^{\mathrm{c}} \ominus\left(W \cap A_{i}^{\mathrm{c}}\right)\right] /\right.$ as if $A_{i}$ in I were perfect $\}$.

Note that the difference between Theorem 3 and Theorem 2 is that the SEs in Theorem 3 are the boundaries of the SEs in Theorem 2; that is, $A_{i}$ and $W \cap \bar{A}_{i}^{\mathrm{c}}$ in the conditions in Theorem 2 become $\frac{A_{i}}{i}$ and $\partial\left(W \cap \bar{A}_{i}^{\mathrm{c}}\right)$. The procedure of proving this theorem follows that of Theorem 2. First, $\tilde{A}_{i} \ominus \partial \tilde{A}_{i} \supseteq \tilde{A}_{i} \ominus \tilde{A}_{i}=(0,0)$ and $\tilde{A}_{i}^{\mathrm{c}} \ominus \partial(W \cap$ $\left.\underline{A_{i}}\right) \supseteq \tilde{A}_{i}^{\mathrm{c}} \ominus\left(W \cap \tilde{A}_{i}\right) \supseteq(0,0)$. Second, for $\tilde{A}_{j}, j \neq i$, either $\bar{A}_{j} \ominus \partial A_{i}=\emptyset$ or $\tilde{A}_{j}^{c} \ominus \partial\left[W \cap \bar{A}_{i}^{c}\right]-\emptyset$. The argument on the size of each cluster which represents the occurrence of an object in $I$ is similar to that corresponding part in Theorem 2, except that here the SEs are the boundary of $A i$ and $\left(W \cap \bar{A}_{i}^{c}\right)$. The detailed proof, which is omitted here, uses the combination of the arguments employed in Theorems 1 and 2.

\section{REMOVAL OF WINDOW IN HIT-OR-MISS TRANSFORMATION}

From the previous sections, the hit-or-miss operators are based on the SEs inside window $W$ for the erosion operation on the complement of input image, $I^{\mathrm{c}}$. The function of the window is to impose a limit on the erosion operation involving the complement of the image, $I^{\mathrm{c}}$. As pointed out in Theorem 1, the boundary of the object, $\left\{\partial A, \partial\left(W \cap A^{c}\right)\right\}$, can be used for structuring elements in the hit-or-miss operation with exactly the same results as if $\left\{A, W \cap A^{c}\right\}$ were used. In Theorems 1,2 , and 3 and the theorems in [3], the object shape $A$ is separated from other shapes in image $I$ by window $W$. The choice of $W$ depends on the object shape $A$, or for every shape $A_{i} \in$ $\mathbf{A}$, select a window $W$ such that $A_{i} \subset W$. For the case in which a shape and the complement of the shape are used as SEs in the hit-or-miss transformation, the window is a necessary portion of the $S E$ for $I^{\mathrm{c}}$, that is, $W \cap A^{\mathrm{c}}$. However, the function of $W$ in separating object $A$ from others becomes trivial when the SEs used in a hit-or-miss transformation are the boundaries of $A$ and $W \cap A^{\mathrm{c}}$.

Let the regions of $A$ and $A^{\mathrm{c}}$ be defined on the plane $Z^{2}$ with each point in $Z^{2}$ corresponding to a point on the Riemann sphere as shown in Fig. 2. The complement $A^{\mathrm{c}}$ of $A$ at infinity is referred to as the point at the top of the Riemann sphere, which does not represent any portion of an image having a finite geometric size. The boundary of $A^{\mathrm{c}}, \partial A^{\mathrm{c}}$, does not include this top point. The notation $\partial A^{\mathrm{c}}$ only refers to the set of points which are the immediate neighboring points of the shape $A$. Note that in topologi-

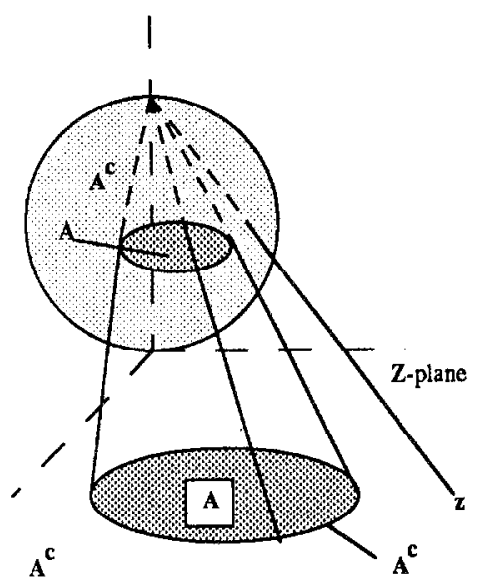

FIG. 2. Illustration of set $A$ represented in domain $Z^{2}$ by an area on a Riemann sphere. The boundary of $A^{\mathrm{c}}$ is defined only on the boundary enclosing $A$.

cal space, $\partial A=\partial A^{\mathrm{c}}=\bar{A} \cap \overline{A^{\mathrm{c}}}$, where $\bar{A}$ is the closure of $A$. In the discrete domain, $\partial A=A \backslash A \ominus \Delta$ and $\partial A^{\mathrm{c}}=$ $A^{\mathrm{c}} \backslash A^{\mathrm{c}} \ominus \Delta$. Figure 3 illustrates the discrete case.

Let $\partial A^{\mathrm{c}}$ be the boundary within $A^{\mathrm{c}}$ between $A$ and $A^{\mathrm{c}}$; then we have that

$\partial A^{\mathrm{CDEF}}=\left\{x: x \in A^{\mathrm{c}}\right.$ and $x$ has at least one immediate neighboring point in $A\}$.

The following theorem can now be formulated.

Theorem 4. Assume that the shape $A$ in $I$ is separated from other shapes $B$ 's, and either $\partial A \nsubseteq B$ or $\partial A^{c} \subseteq$ $B^{c} ;$ then the shape A occurs in the image I at, and only at, the locations represented by set $L$,

$$
L=[I \ominus \partial A] \cap\left[I^{\mathfrak{c}} \ominus \partial A^{\mathfrak{c}}\right] .
$$

Proof. First to prove two preliminarics: (a) $(A \ominus \partial A)$ $\cap\left(A^{\mathrm{c}} \ominus \partial A^{\mathrm{c}}\right)=\{(0,0)\} ;$ and (b) $(B \ominus \partial A) \cap\left(B^{\mathrm{c}} \ominus \partial A^{\mathrm{c}}\right)=\emptyset$ for $B \neq A$.

To prove (a), we already know from Lemma 2 that $A \ominus$ $\partial A=\{(0,0)\}$. For $x \in A^{\mathrm{c}} \ominus \partial A^{\mathrm{c}}$, then $x+\partial A^{\mathrm{c}} \subseteq A^{\mathrm{c}}$. For $x=(0,0),(0,0)+\partial A^{\mathrm{c}} \subseteq A^{\mathrm{c}}$ is true. Therefore, $(A \ominus \partial A) \cap$ $\left(A^{\mathrm{c}} \ominus \partial A^{\mathrm{c}}\right)=\{(0,0)\} \cap\left\{(0,0) \cup\left[\left(A^{\mathrm{c}} \ominus \partial A^{\mathrm{c}}\right) \backslash(0,0)\right]\right\}=$ $\{(0,0)\}$.

'lo prove (b), for $B \neq A$, three possible situations need to be considered: (1) There exists an $x$ such that $B_{x} \subset A$. From Lemma 1, $(B \Theta \partial A)_{x}=B_{x} \ominus \partial A=\emptyset$. (2) There exists an $x$ such that $B_{x} \supset A$. Then, $B \ominus \partial A \neq \emptyset$. Assume that $z \in B \ominus \partial A \subseteq B_{a / \forall a \in \partial A}$. For some $a \in A, B_{a} \subseteq B \oplus \partial A$ $\subseteq B \oplus \partial A^{\mathrm{c}}$ because $B_{x} \supset A$. Hence $z \in B \ominus \partial A \Rightarrow z \in B \oplus$ $\partial A^{\mathrm{c}}$, and this is a contradiction to $z \in B^{\mathrm{c}} \ominus \partial A^{\mathrm{c}}=(B \oplus$ $\left.\partial A^{\mathrm{c}}\right)^{\mathrm{c}}$. (3) There does not exist an $x$ such that $B_{x} \subset A$ or 

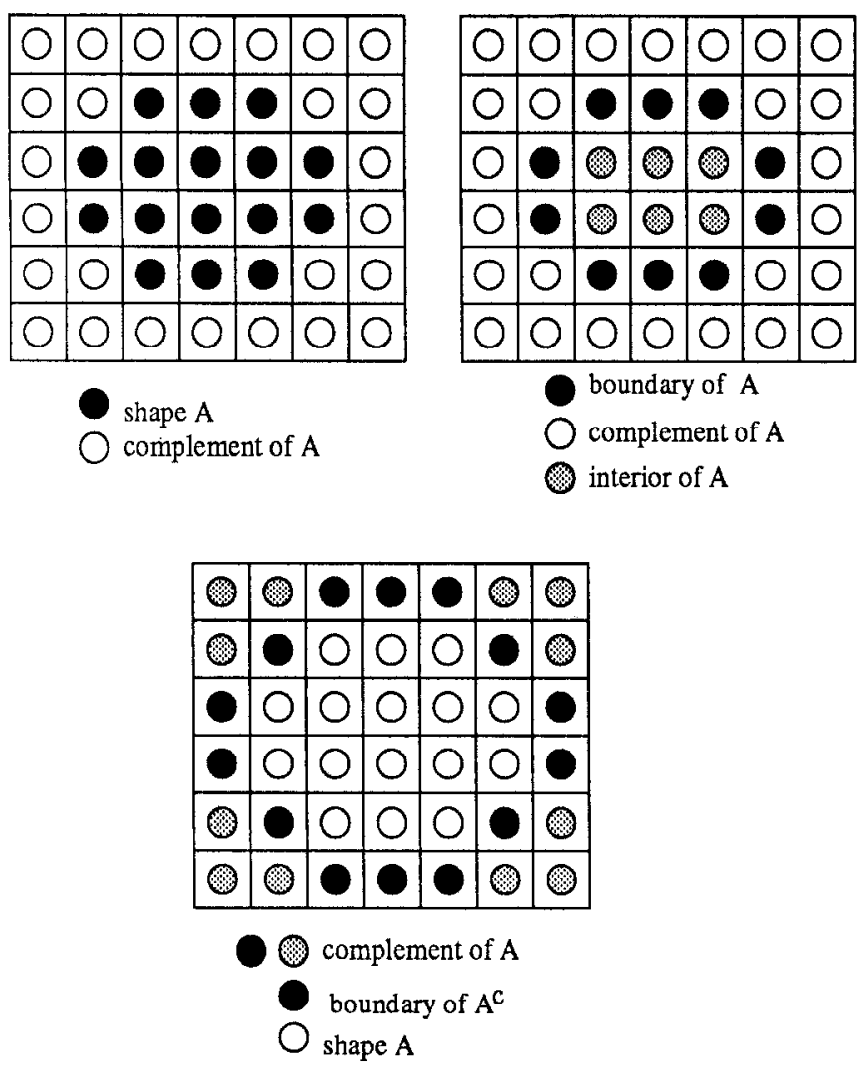

FIG. 3. Illustration of shape, boundary of shape, and boundary of complement of shape.

$B_{x} \supset A$, but for some $x, B_{x} \cap A \neq \emptyset$. In this case, $B \ominus \partial A$ $=\left(B_{x} \ominus \partial A\right)_{-x}=\emptyset$ according to Lemma 1 .

Let $S(k) \in \mathbf{S}$ and $A \in \mathbf{S}$, where $\mathbf{S}$ is the sample space. Then for $S(k) \neq A,\left(S(k)_{p_{k}} \ominus \partial A\right) \cap\left(S(k)_{p_{k}}^{\mathrm{c}} \ominus \partial A^{\mathrm{c}}\right)=[(S(k)$ $\left.\ominus \partial A) \cap\left(S(k)^{\mathrm{c}} \ominus \partial A^{\mathrm{c}}\right)\right]_{p_{k}}=\emptyset$. Assume that all shapes $\{S(k)\}$ in $I$ are separated and $A$ appears in $I$ at $\left\{p_{k}\right\}$; then

$$
\begin{aligned}
& (I \ominus \partial A) \cap\left(I^{\mathrm{c}} \ominus \partial A^{\mathrm{c}}\right) \\
= & (I \ominus \partial A) \cap\left(I \oplus \partial A^{\mathrm{c}}\right)^{\mathrm{c}} \\
= & {\left[\left(\bigcup_{k} S(k)_{p_{k}}\right) \ominus \partial A\right] \cap\left[\left(\bigcup_{k} S(k)_{p_{k}}\right) \oplus \partial A^{\mathrm{c}}\right]^{\mathrm{c}} } \\
= & {\left[\bigcup_{k}\left(S(k)_{p_{k}} \ominus \partial A\right)\right] \cap\left[\bigcup_{k}\left(S(k)_{p_{k}} \oplus \partial A^{\mathrm{c}}\right)\right]^{\mathrm{c}} } \\
= & {\left[\bigcup_{k}\left(S(k)_{p_{k}} \ominus \partial A\right)\right] \cap\left[\bigcap_{k}\left(S(k)_{p_{k}}^{\mathrm{c}} \oplus \partial A^{\mathrm{c}}\right)\right] } \\
= & \bigcup_{k}\left[\left(S(k)_{p_{k}} \ominus \partial A\right) \cap\left(S(k)_{p_{k}}^{\mathrm{c}} \ominus \partial A^{\mathrm{c}}\right)\right. \\
& \left.\bigcap_{l \neq k} S(l)_{P_{l}}^{\mathrm{c}} \ominus \partial A^{\mathrm{c}}\right]
\end{aligned}
$$

$$
\begin{aligned}
= & \left\{\bigcup _ { k ^ { \prime } } \left[\left(A_{p_{k^{\prime}}} \ominus \partial A\right) \cap\left(A_{p_{k^{\prime}}}^{\mathrm{c}} \ominus \partial A^{\mathrm{c}}\right)\right.\right. \\
& \left.\left.\bigcap_{l \neq k} S(l)_{\mathrm{p}_{l}}^{\mathrm{c}} \ominus \partial A^{\mathrm{c}}\right]\right\} \bigcup_{S(k) \neq A} \\
& \left\{\bigcup _ { k } \left[\left(S(k)_{p_{k}} \ominus \partial A\right) \cap\left(S(k)_{p_{k}}^{\mathrm{c}} \ominus \partial A^{\mathrm{c}}\right)\right.\right. \\
& \left.\left.\bigcap_{l \neq k} S(l)_{p_{l}}^{\mathrm{c}} \ominus \partial A^{\mathrm{c}}\right]\right\} \\
= & \bigcup_{k^{\prime}}\left[\left(A_{p_{k^{\prime}}} \ominus \partial A\right) \cap\left(A_{p_{k^{\prime}}}^{\mathrm{c}} \ominus \partial A^{\mathrm{c}}\right) \bigcap_{l+k} S(l)_{p_{l}}^{\mathrm{c}} \ominus \partial A^{\mathrm{c}}\right] \\
= & \bigcup_{k^{\prime}}\left[\left(A_{p_{k^{\prime}}} \ominus \partial A\right) \cap\left(A_{p_{k^{\prime}}}^{\mathrm{c}} \ominus \partial A^{\mathrm{c}}\right)\right] \\
= & \bigcup_{k^{\prime}}\left\{p_{k^{\prime}}\right\} .
\end{aligned}
$$

Equation (50) follows from Lq. (49) since $A_{p_{k^{\prime}}}$ is separated from $S(l)_{p_{l}}$. This indicates that $x \notin S(l) \oplus \partial A^{\mathrm{c}}$ for $x \in\left[A_{p_{k^{\prime}}}, \ominus \partial A\right] \cap\left[A_{p_{k^{\prime}}}^{\mathrm{c}} \ominus \partial A^{\mathrm{c}}\right]$. As a consequence, $x \in$ $\left(S(l)_{p_{l}} \oplus \partial A^{\mathrm{c}}\right)^{\mathrm{c}}=S(i)_{\mathrm{p}_{1}}^{\mathrm{c}} \ominus \partial A^{\mathrm{c}}$.

Q.E.D.

Without restrictions on the choice of window $W$, the hit-or-miss transformation can readily be used as a practical tool for automated shape recognition. The use of a window in the erosion of $I^{\mathrm{c}}$ by $\left(W \cap A^{\mathrm{c}}\right.$ ) could prevent the hit-or-miss transformation from successfully locating the target object if there are some other objects close to it, such as portions of other objects falling within the window. When window $W$ is removed from the hit-or-miss transformation, the areas of interest around the target object $A$ are essentially the boundaries of the shape, $\partial A$ and $\partial A^{c}$. Figure 4 illustrates a situation wherein nearby objects are very close to the object of interest and use of window $W$ becomes inappropriate.

In addition to the advantage of increased flexibility brought by removal of restrictions on window choice, the process also achieves additional savings in the hit-ormiss operation because all frame shiftings and logic AND
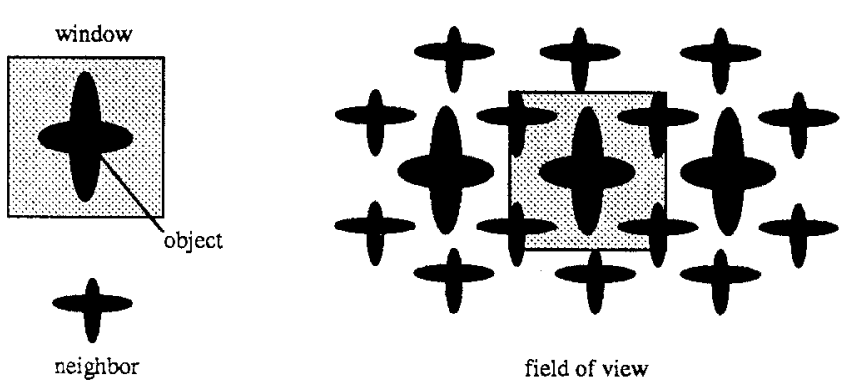

FIG. 4. Unacceptable choice of window due to the close proximity of neighboring objects. 
operations are not performed for all points on the boundary of window $W$.

In recognizing indeterminate variations of an object shape, the role of the window is the same as that when it is used in the case of recognizing perfect shapes. The details in need of refinement in Theorem 3 are those related to the window $W$. Without use of a window, the process of recognition is principally the same as that stated in Theorem 3.

Theorem 5. Let $A_{i}$ be a shape in the sample space $\mathbf{A}$, $\tilde{A}_{i}$ be an indeterminate variation of $A_{i}$ in image $I$ where $\left\{\tilde{A}_{i}, I\right\} \in Z^{2}, A_{i}$ be the lower bound of variations of shape $A_{i}$, and $\bar{A}_{i}$ be the upper bound of variations of shape $A_{i}$. $\left(A_{i}\right)_{p_{k}}$ is $A_{i}$ within image I at location $p_{k}$ is separated from other shapes in I by $\bar{A}_{i}$; that is, $\left(\bar{A}_{i}\right)_{p_{k}} \cap\left(\tilde{A}_{j}\right)_{p_{l}}=\emptyset$ for any $A \in A$ and $p_{l} \neq p_{k}$. If the following conditions are satisfied,

(1) $\partial \underline{A}_{i} \subseteq \tilde{A}_{i}$ and $\partial \bar{A}_{i}^{\mathrm{c}} \subseteq \tilde{A}_{i}^{\mathrm{c}}$,

(2) for any $x, \partial \underline{A_{i}}+x \underline{\Phi} \tilde{A}_{j}$ or $\partial \bar{A}_{i}^{\mathrm{c}}+x \subseteq \tilde{A}_{j}^{\mathrm{c}}$ for $j \neq i$,

then, $\tilde{A}_{i}$, hence $A_{i}$, is at location $p$, where

$$
p \in\left[I \ominus \partial \underline{A_{i}}\right] \cap\left[I^{\mathrm{c}} \ominus \partial \bar{A}_{i}^{\mathrm{c}}\right] \subseteq \bigcup_{q \in Q} E_{q}
$$

while $E=\frac{1}{2}\left(\bar{A}_{i} \ominus \underline{A_{i}}\right)$ and $Q=\left\{z: z \in\left[I \ominus A_{i} \cap I^{\mathrm{c}} \ominus(W \cap\right.\right.$ $\left.\left.A_{i}^{\mathrm{c}}\right)\right] /$ as if $A_{i}$ in I were perfect $\}$.

Proof. From condition (1), $\bar{A}_{i} \ominus \partial A_{i} \supseteq \bar{A}_{i} \ominus \bar{A}_{i}=\{(0,0)\}$ and $\tilde{A}_{i}^{\mathrm{c}} \ominus \partial \bar{A}_{i}^{\mathrm{c}} \supseteq \tilde{A}_{i}^{\mathrm{c}} \ominus \bar{A}_{i}^{\mathrm{c}}=\{(0,0)\}$. Hence, $\tilde{A}_{i} \ominus \partial A_{i} \cap \tilde{A}_{i}^{\mathrm{c}}$ $\ominus \partial \bar{A}_{i}^{i} \supseteq\{(0,0)\}$. From condition (2), $\tilde{A}_{j} \ominus \partial \underline{A_{i}}=\left(\left(\tilde{A}_{j}\right)_{x} \ominus\right.$ $\left.\partial \underline{A_{i}}\right)_{-x}=\emptyset$ if $\partial \underline{A}_{i}+x \nsubseteq \tilde{A_{j}}$. Furthermore, we have that $\tilde{A}_{j}^{\mathrm{c}}$ $\widehat{\ominus \partial} \bar{A}_{i}^{\mathrm{c}}=\left(\left(\tilde{A}_{j}^{\mathrm{c}}\right)_{x} \ominus \partial \bar{A}_{i}^{\mathrm{c}}\right)_{-x}=\emptyset$ if $\partial \bar{A}_{i}^{\mathrm{c}}+x \underline{A_{\mathrm{A}}} \tilde{A}_{j}^{\mathrm{c}}$. The resulting cluster of points $\left(\tilde{A}_{i} \ominus \partial A_{i}\right) \cap\left(\tilde{A}_{i}^{c} \ominus \partial \bar{A}_{i}^{c}\right)$ should be within $E=\frac{1}{2}\left(\overline{A_{i}} \ominus A_{i}\right)$, according to the arguments presented in Theorem 2. Hence, the resultant set from the hit-or-miss transformation is obtained as follows:

$$
\begin{aligned}
& {\left[I \ominus \partial \underline{A_{i}}\right] \cap\left[I^{\mathrm{c}} \ominus \partial \bar{A}_{i}^{\mathrm{c}}\right] } \\
= & {\left[I \ominus \partial \underline{A_{i}}\right] \cap\left[I \oplus \partial \bar{A}_{i}^{\mathrm{c}}\right]^{\mathrm{c}} } \\
= & {\left[\left(\bigcup_{k} \tilde{S}(k)_{p_{k}}\right) \ominus \partial \underline{A_{i}}\right] \cap\left[\left(\bigcup_{i} \tilde{S}(l)_{p_{l}}\right) \oplus \partial \bar{A}_{i}\right]^{\mathrm{c}} } \\
= & {\left[\bigcup_{k}\left(\tilde{S}(k)_{p_{k}} \ominus \partial \underline{A_{i}}\right)\right] \cap\left[\bigcup_{l}\left(\tilde{S}(l)_{p_{l}} \oplus \partial \underline{A_{i}}\right)\right]^{\mathrm{c}} } \\
= & {\left[\bigcup_{k}\left(\tilde{S}(k)_{p_{k}} \ominus \partial \underline{\left.A_{i}\right)}\right] \cap\left[\bigcap_{l}\left(\tilde{S}(l)_{p_{i}}^{\mathrm{c}} \ominus \partial \bar{A}_{i}^{\mathrm{c}}\right)\right]\right.} \\
= & \bigcup_{k}\left[\left(\tilde{S}(k)_{p_{k}} \ominus \underline{\partial A_{i}}\right) \cap\left(\tilde{S}(k)_{p_{k}}^{\mathrm{c}} \ominus \partial \bar{A}_{i}^{\mathrm{c}}\right)\right. \\
& \left.\bigcap_{p_{i} \neq p_{k}}\left(\tilde{S}(l)_{p_{l}}^{\mathrm{c}} \ominus \partial \bar{A}_{i}^{\mathrm{c}}\right)\right]
\end{aligned}
$$

$$
\begin{aligned}
& =\left\{\bigcup_{k^{\prime}}\left[\left(\tilde{A}_{i_{p_{k}}} \ominus \partial \underline{A_{i}}\right) \cap\left(\tilde{A}_{i_{p_{k}}}^{\mathrm{c}} \ominus \partial \bar{A}_{i}^{\mathrm{c}}\right)\right]\right. \\
& \left.\bigcap_{p_{l} \neq p_{k^{\prime}}}\left(\tilde{S}(l)_{p_{l}}^{\mathrm{c}} \ominus \partial \bar{A}_{i}^{\mathrm{c}}\right)\right\} \\
& \cup\left\{\bigcup_{S(k) \neq A_{i}}\left[\left(\tilde{S}(k)_{p_{k}} \ominus \partial \underline{A_{i}}\right) \cap\left(\tilde{S}(k)_{p_{k}}^{\mathrm{c}} \ominus \partial \bar{A}_{i}^{\mathrm{c}}\right)\right]\right. \\
& \left.\bigcap_{p_{i} \neq p_{k}}\left(\tilde{S}(l)_{p_{l}}^{c} \ominus \partial \bar{A}_{i}^{c}\right)\right\} \\
& =\bigcup_{k^{\prime}}\left[\left(\tilde{A}_{i_{p^{\prime}}} \ominus \partial \underline{\partial A_{i}}\right) \cap\left(\tilde{A}_{i_{p^{\prime}}}^{\mathrm{c}} \ominus \partial \overline{A_{i}^{\mathrm{c}}}\right)\right. \\
& \left.\bigcap_{p_{i} \neq p_{k^{\prime}}}\left(\tilde{S}(l)_{p_{l}}^{c} \ominus \partial \bar{A}_{i}^{c}\right)\right] \\
& =\bigcup_{k^{\prime}}\left[\left(\tilde{A}_{i_{p k^{\prime}}} \ominus \partial \underline{A_{i}}\right) \cap\left(\tilde{A}_{i_{p k^{\prime}}}^{\mathrm{c}} \ominus \partial \bar{A}_{i}^{\mathrm{c}}\right)\right] \\
& \subseteq \bigcup_{k^{\prime}} E_{p_{k^{\prime}}} .
\end{aligned}
$$

Equation (57) results in Eq. (58) due to the fact that $x \notin$ $\tilde{S}(l)_{p_{l}} \oplus \partial \bar{A}_{i}^{\mathrm{c}}$ since $\tilde{S}(l)_{p_{l}}$ is separated from $\tilde{A}_{i_{p_{t}}}$. Consequently, this means $x \in\left[\tilde{S}(l)_{p_{l}} \oplus \partial \bar{A}_{i}^{\mathrm{c}}\right]^{\mathrm{c}}=\tilde{S}(l)_{p_{l}}^{\mathrm{c}} \ominus \partial \bar{A}_{i}^{\mathrm{c}}$.

Q.E.D.

Structuring elements used without a window also provide more flexibility in the recognition of indeterminate variations of an object shape, compared to the case wherein SEs are used with a window. This is so because the range of variation in the shape of an object can be extended from $W \cap \tilde{A}_{i}$ to $\overline{A_{i}} \cap \partial \bar{A}_{i}^{\mathrm{c}}$ which does not intersect with other possible shapes.

\section{EXAMPLES}

We have presented an algorithm in which the scts rcpresenting the structuring elements are reduced to the boundaries of the corresponding object. We claim that by doing so, the inherent redundancy in the hit-or-miss transformation is reduced. Further, we discussed the removal of window restrictions. An example is given here to illustrate Theorem 3. In Fig. 5, the shape " $\mathrm{H}$ " is tested for the range of its variation and the computation time is compared between the cases in which the shape boundary is used as a structuring element and in which the shape itself is used as a structuring element. Figures 5(a1)-(a6) show the inward variation of the shape " $H$ ". That is, the variation of " $H$ " is presented as a shrunken version of the original shape. The black area in each figure is the area in which the variation of that shape is allowed. The white line between the black and the gray areas is used as the structuring element. The gray area is the interior of the shape which is excluded from being a part of the structuring element. Figures 5(b1)-(b6) show the outward variation of the object, that is, the swelled 


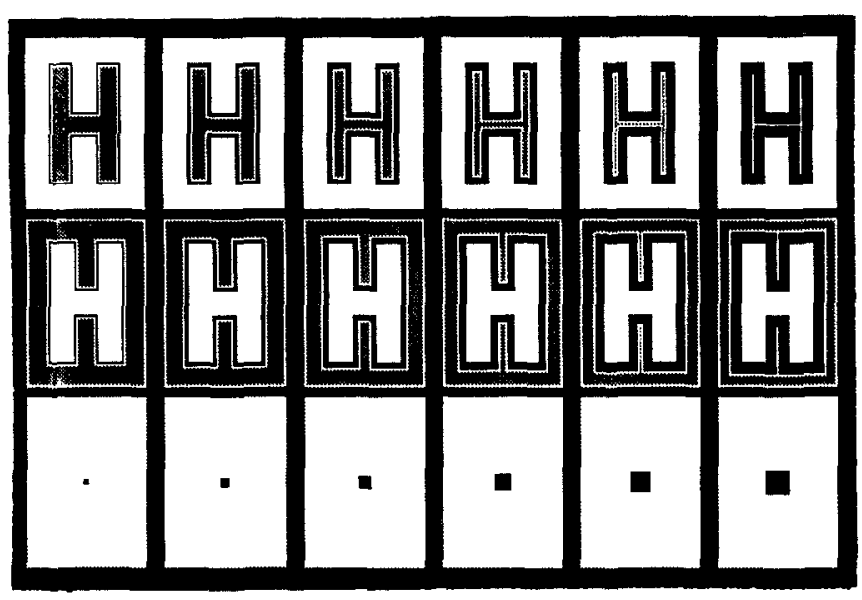

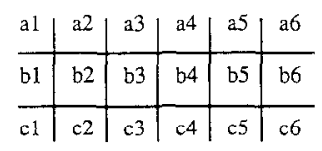

FIG. 5. The variations of shape " $\mathrm{H}$ " and the results of the bit-ormiss transformations. (a1-a6) Inward variation; the black area is the allowed range of variation; the white lines are the boundary of $\tilde{A}$; gray areas indicate where redundancy is present in the hit-or-miss transformation. (b1-b6) Outward variation on the complement of shape. (cl-c6) Set of location within a predetermined circle. version of the original object. The black arca is the cstimated range of variation. The white line between the black and the gray area is used as the strucluring element for the erosion operation on the complement of the shape. The gray area is the interior within the window and is excluded from being a part of the SE in accordance with Theorem 3 . Figures $5(\mathrm{c} 1)-(\mathrm{c} 6)$ show the result of the hit-or-miss transformation. As we noted earlier, the area of the resulting sets of locations becomes larger as the variations of the shape become larger.

For this example, the ratio of the varied shape size over the original shape size is illustrated in Fig. 6a. Comparisons are made between the variation of the shape and the original, as well as between the variation of the windowed complement of the shape and the complement of the original shape. Figure $6 \mathrm{~b}$ shows the processing time required when only the boundary is used as a structuring element versus that for when the shape itself is used as a structuring element. Figure $6 \mathrm{c}$ shows the ratio of processing time required for the hit-or-miss transformation without window restriction and using the boundary of the shape over that using the shape itself as structuring elcment as described by Theorem 5 . shape variation ratio: variation/shape

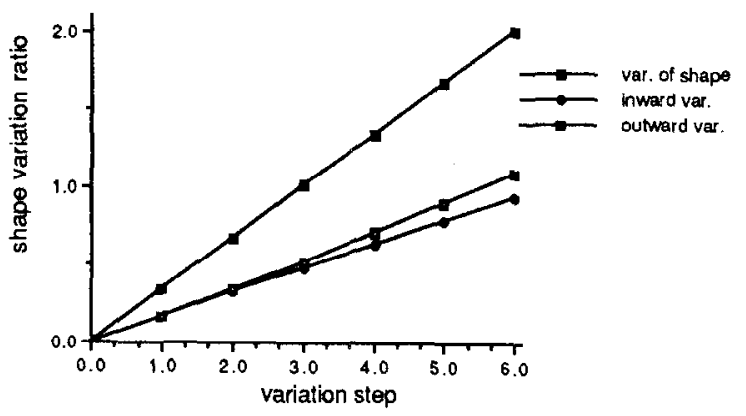

(a)

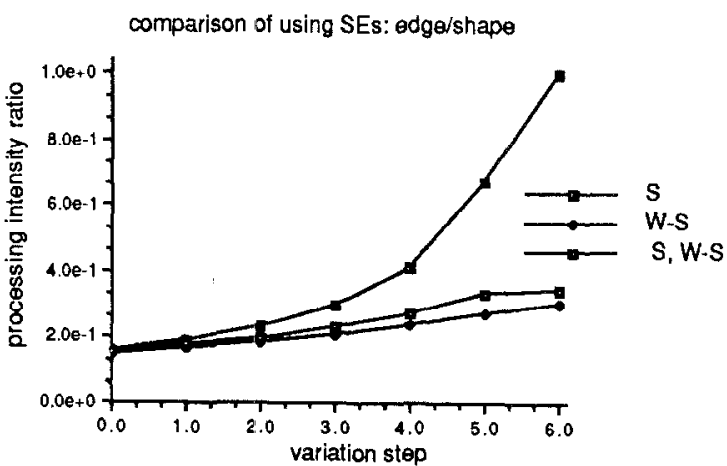

(b)

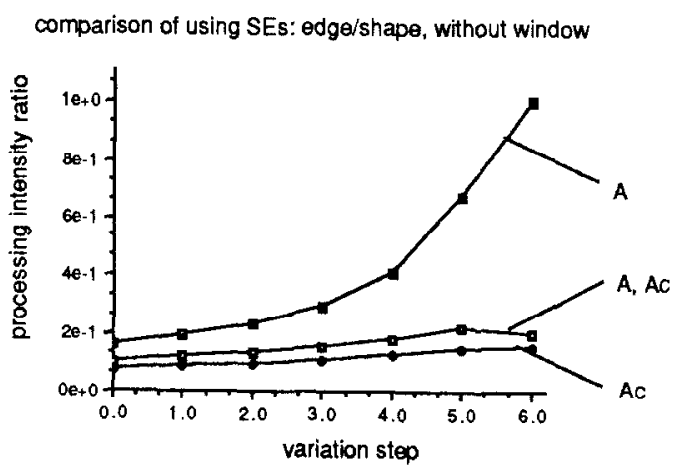

(c)

FIG. 6. Statistics for the example shown in Fig. 5. (a) Ratio of areas of variation over the areas of the original shape. (b) Ratio of computation intensity when using the boundary of a shape as an SE over that when using the shape itself as an SE. (c) Ratio of computation intensity using the boundary of a shape as an SF in the hit-or-miss transformation without window $W$ over that when using the shape itself as an SE. 


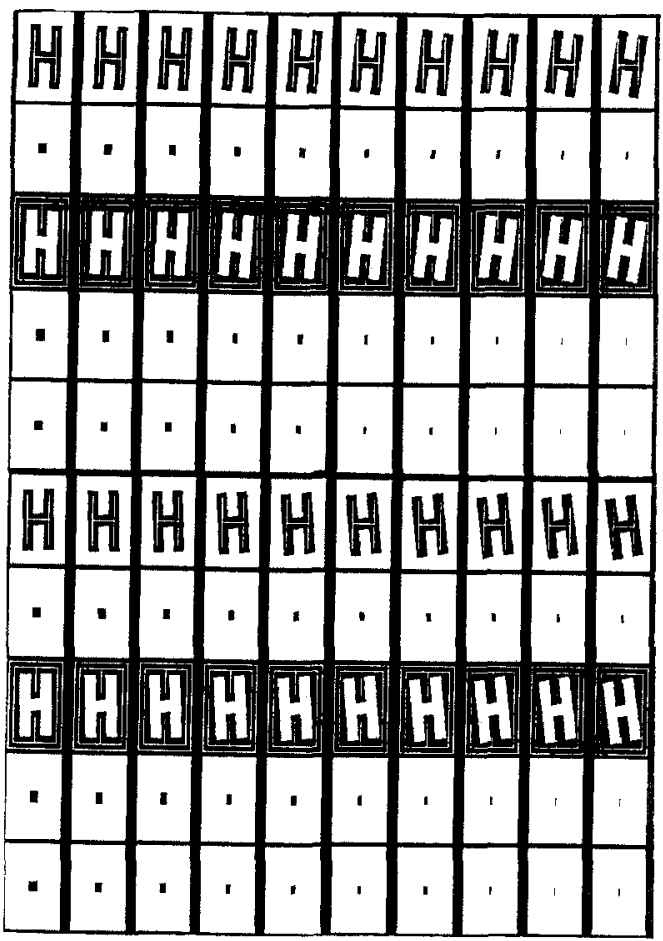

(b)

FIG. 7. Matching of the object $H$ with tilted versions of $H$. (a) The hit-or-miss transformation for untilted $I I$ and the SEs. (b) The hit-ormiss transformation for tilted $H$ 's with predefined SEs shown in (a) as the white lines.

Figure 7 shows another example wherein Theorem 3 is used to match an object shape with the varied acquired shapes. The object shape is an $H$, shown in the top block of Fig. 7a. The SE for $H$ is the skeleton of $H, \operatorname{SK}(H)$, the white lines shown in the second block of Fig. 7a. From skeletonization $[1,5]$, we know that $\operatorname{SK}(H) \subset H$. The SE for $H^{\mathrm{c}}, \partial\left(W \cap \bar{H}^{\mathrm{c}}\right)$, is the skeleton of $W \cap H^{\mathrm{c}}$ and the edge of $W$, shown in the fourth block of Fig. 7a. The third block of Fig. 7a shows the resulting set $H \ominus \mathrm{SK}(H)$ and the fifth block shows the resulting set $H^{\mathrm{c}} \ominus \partial\left(W \cap \bar{H}^{\mathrm{c}}\right)$. The sixth block displays the resulting set of $[H \ominus \mathrm{SK}(H)]$ $\cap\left[H^{\mathrm{c}} \ominus \partial\left(W \cap \bar{H}^{\mathrm{c}}\right)\right]$. Figure $7 \mathrm{~b}$ shows the process of matching tilted $H$ shapes, where the first row shows that $H$ is tilted toward the right by $1^{\circ}$ to $10^{\circ}$ and the sixth row shows that $H$ is tilted toward the left by $1^{\circ}$ to $10^{\circ}$. The third and eighth rows show the windowed complements of the tilted $H$ where it is tilted toward the right and the left, respectively. The SEs are white lines inside the tilted $H$ 's and they are the same as those shown in Fig. 7a, respectively. The fifth and tenth rows show the sets resulting from the hit-or-miss transformations.

The next example shows a character recognition pro- cess using the hit-or-miss transformation suggested in Theorem 3. A gray-scale image is properly thresholded into a binary image as shown in Fig. 8a. The shape used for constructing an $\mathrm{SE}$ is shown in the upper-right corner, which is the character " $n$ ". Here, the SE $\partial A$ is the boundary of the shrunken version of " $n$ " by erosion. This $\mathrm{SE}$ is inputted to the erosion operation $[I \ominus \partial A]$. The foreground (black) in Fig. 8b represents the occurrence of " $n$ " as well as the shapes which can enclose the letter " $n$ ", e.g., " $m$ ". Note that the letter " $m$ " shown in $I$ approximates two combined " $n$ " letters; therefore, the erosion operation reflects their presentation. In Fig. 8c, the foreground (black) is the result of erosion [ $I^{\mathrm{c}} \ominus$ $\left.\partial\left(W \cap \bar{A}^{\mathrm{c}}\right)\right]$ where the $\mathrm{SE}, \partial\left(W \cap \bar{A}^{\mathrm{c}}\right)$ of windowed complement of the shape " $n$ ", is shown in the upper-right corner. The complement of letter " $m$ " does satisfy the second term of Condition (2) in Theorem 3. Specifically, the boundary of the windowed complement of " $n$ " after eroding is not included in the windowed complement of " $\mathrm{m}$ ". Therefore, the place corresponding to " $\mathrm{m}$ " exhibits the background that dismisses a possible detection of " $m$ " as " $n$ ". The result of the hit-or-miss operation is shown in Fig. 8d. In this example, the operation employed on the shape " $n$ " to transform it into an SE is that of an erosion. The same operation is performed on the windowed complement of " $n$ " to obtain the SE for eroding the complement of the image. One advantage of applying erosion on the shape " $n$ " to construct an SE is that the predicted shape variation can be either enlarged or reduced. The erosion operation translates $\left(A, W \cap A^{\mathrm{c}}\right)$ into $\left(A, W \cap \bar{A}^{\mathrm{c}}\right)$ which can satisfy the first condition of the theorems in Section 3.

The characters in the images of printed text are generally uniform in their width. The connectivities are apparent among any portion of a character, except for a few cases like lowercase $i, j$. This observation suggests that a skeletonization can be employed to represent the SE. When using the skeleton as an SE, a maximal range of variation is available when meeting the first condition of the theorems. Also, use of the skeleton still preserves the characteristics of that shape, as illustrated in Fig. 9. The object to be recognized is the letter " $n$ " shown in Fig. 9a. The recognition is again successful as shown in Figs. 9a$9 \mathrm{~d}$. From this example, we can see that using the skeleton of " $n$ " as the SE yields a reasonable range of flexibility for the hit-or-miss operation. The skeleton of character " $n$ " is used as SE for the operation $I \ominus A$, where $A$ is the skeleton of " $n$ ". In this example, the use of the skeleton is appropriate because the object shape is thin. To satisfy the condition $A \subseteq \tilde{A}$ in Theorem 5, $A$ must be chosen such that $A$ can bc fit inside all possible appearances of " $n$ ". The medial axis of the object character " $n$ " apparently mects the rcquirement; hence the skeletonization algorithm $[1,5]$ is employed in obtaining such an SE. 


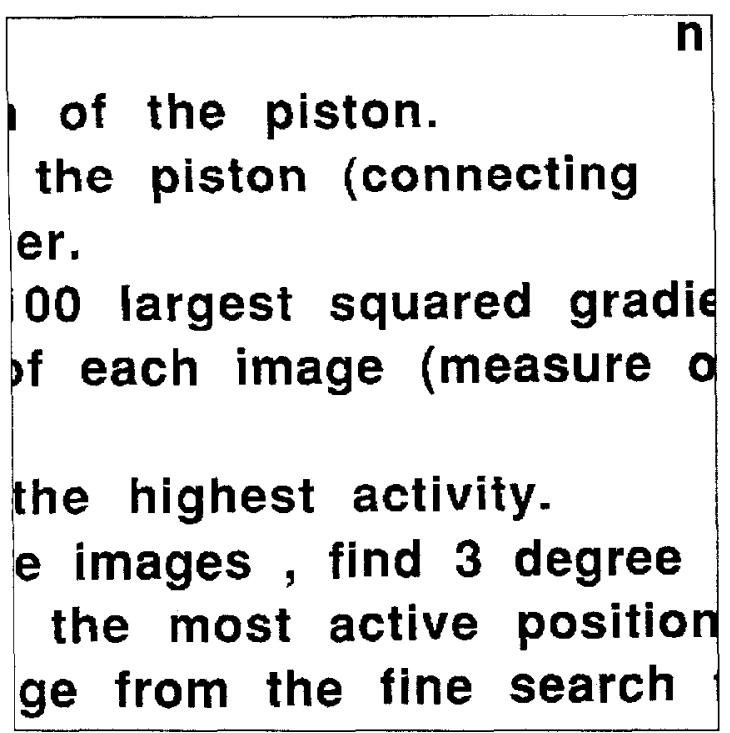

(a)

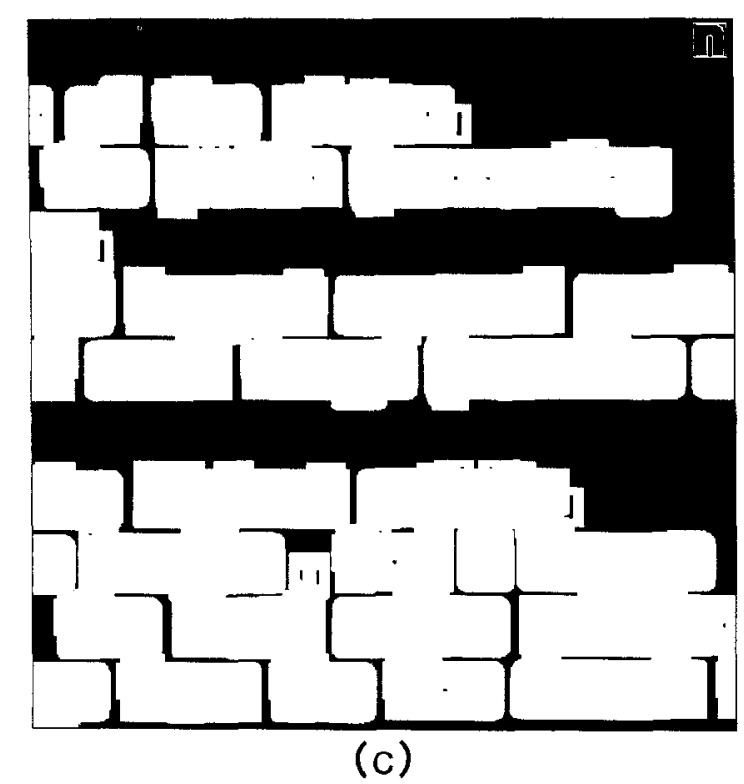

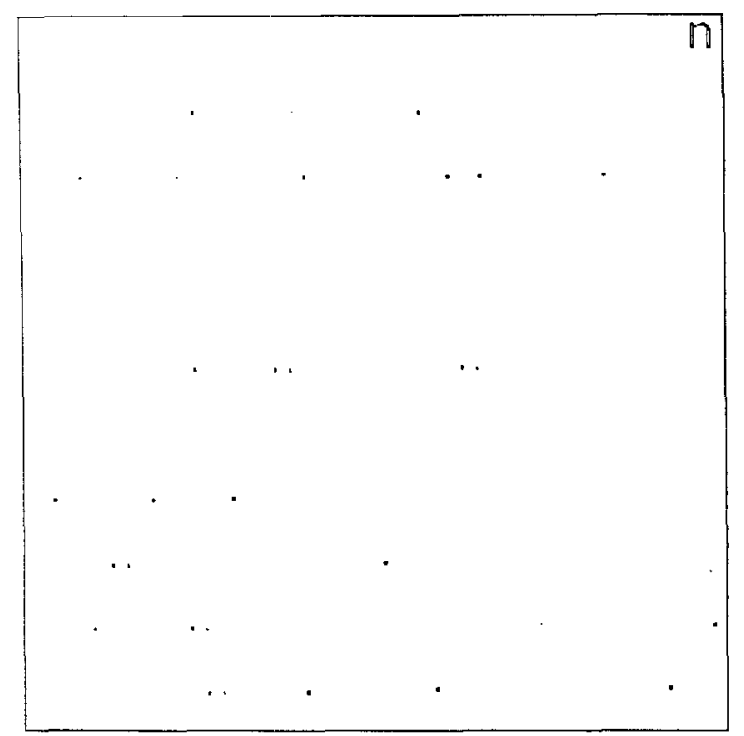

(b)

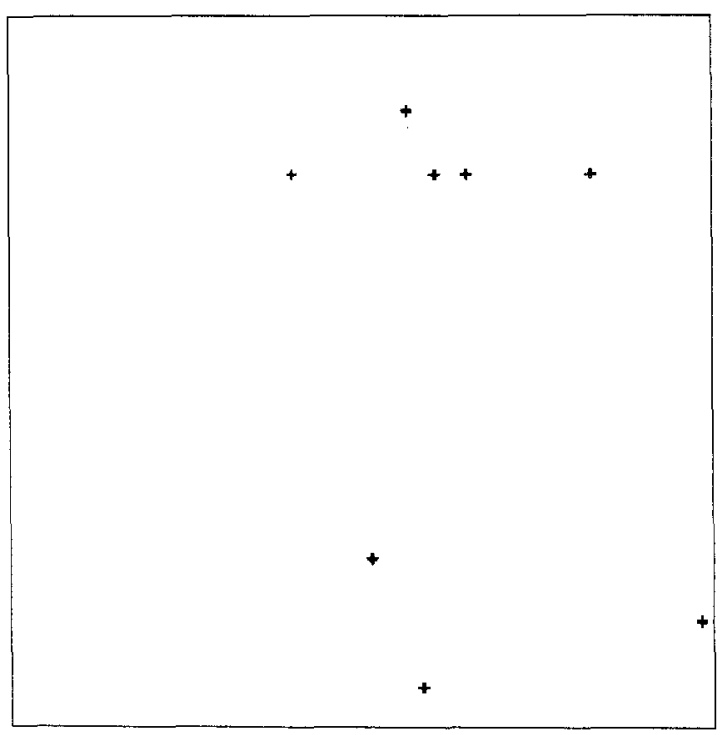

(d)

FIG. 8. Recognition of English letter " $n$ " in the binary image. (a) Binary image obtained from thresholding a text image; the " $n$ " shown in the upper-right corner is used to construct the SE. (b) Result of eroding the image of (a) using the boundary of an eroded " $n$ " as SE. (c) Result of eroding the complement of the image (a) using the boundary of an eroded $W \cap A^{\mathrm{c}}, A=$ " $\mathrm{n}$ ", as the SE. (d) Result of AND operation between images (b) and (c), with each occurrence of " $n$ " marked by a cross.

\section{CONCLUSIONS}

Shape recognition using morphological operators provides a new approach to the process of automated object identification. For perfect shapes, a new theorem applied to the shape recognition problem has been presented. This theorem presents an alternative to an existing approach [3] using a morphological hit-or-miss transformation and indicates a clear advantage in the reduction of redundancy involved in the matching process where morphological operations are used.

In the case of imperfect shapes, a theorem for locating a desired shape which has changed indeterminately from the a priori object shape is provided. The SEs used in these processes are the boundaries of the shapes which correspond to the sample shapes and take account of indeterminate variations. This method does not require an exhaustive investigation of all possible variations of a 


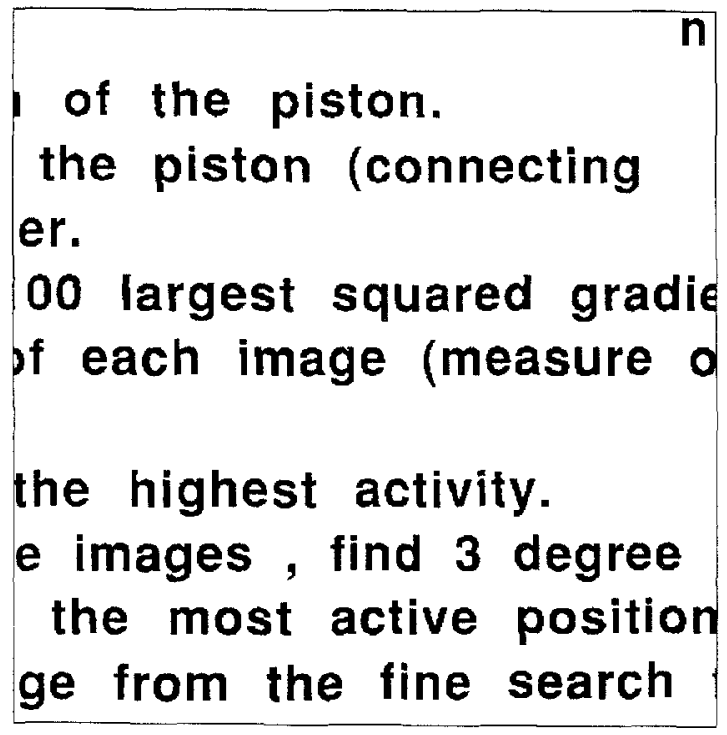

(a)

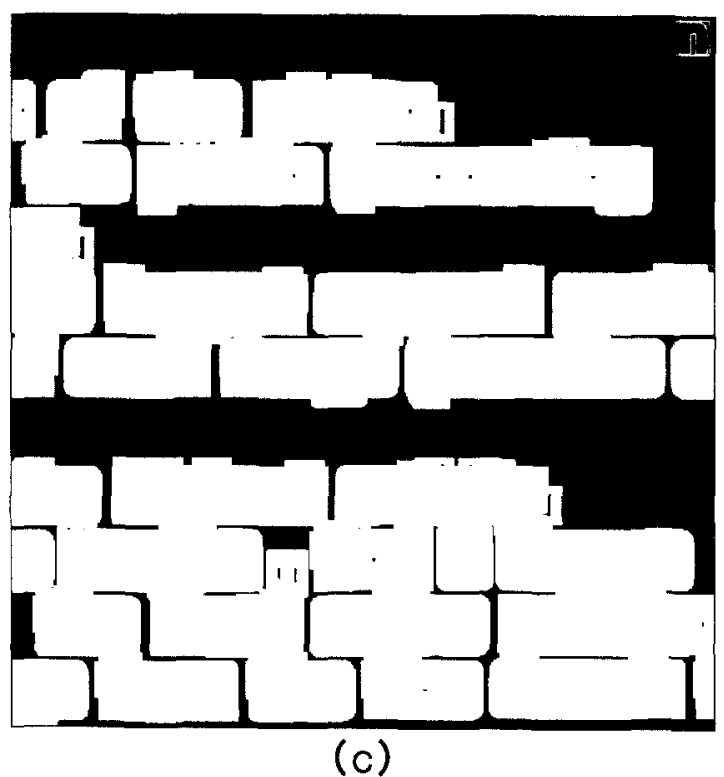

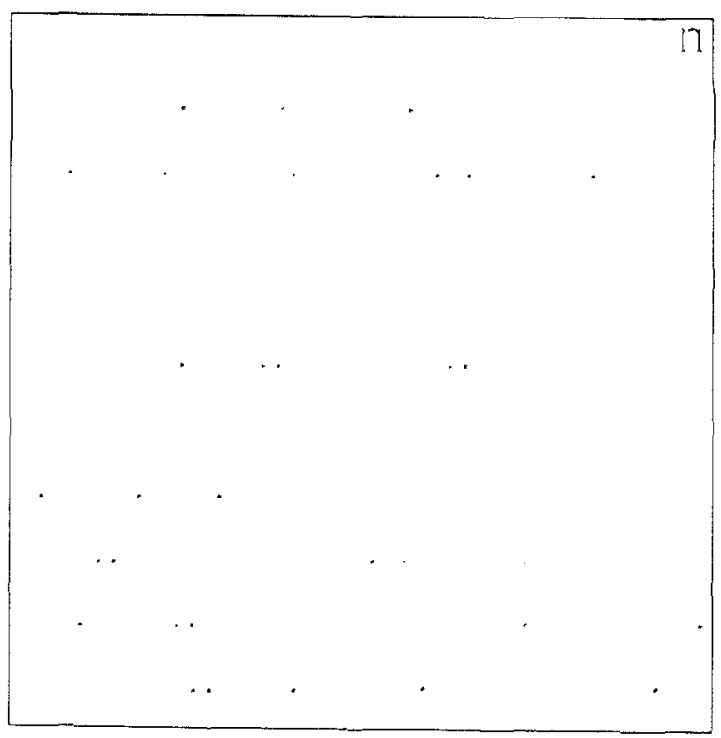

(b)

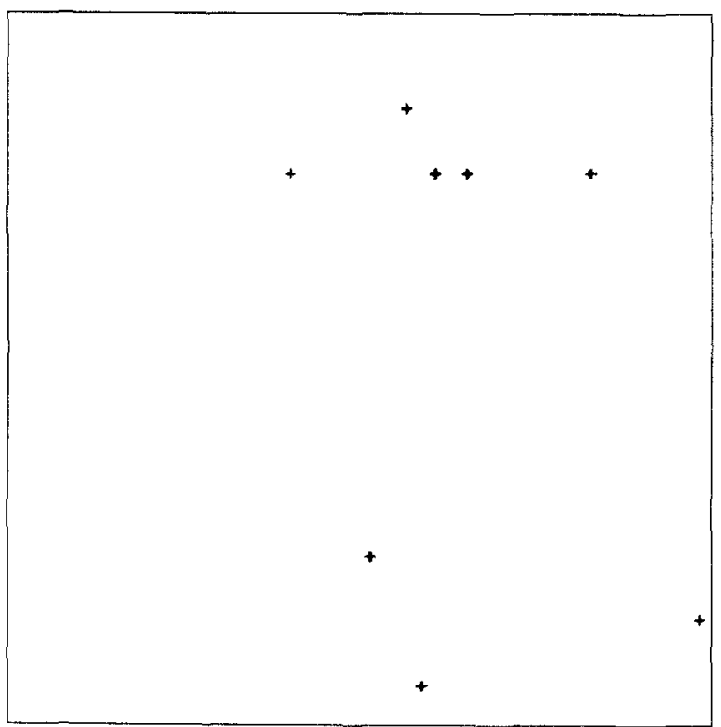

(d)

FIG. 9. Recognition of English letter " $n$ " in the binary image using skeleton of " $n$ " as SE. (a) Binary image. (b) Result of eroding image (a) using the skeleton of " $n$ " shown in the upper-right corner of (a) as the SE. (c) Result of eroding the complement of image (a) using an eroded $W \cap A^{c}, A=$ " $n$ ", as the SE. (d) Result of AND operation between images (b) and (c) with each occurrence of " $n$ " marked by a cross.

shape contained in the real-world image. Hence, we have developed a practical approach to solve the problem of matching desired shapes in noisy images where the observed shapes are imperfect. The shape boundaries are used as SEs. The conditions for recognizing imperfect shapes are relatively easy to satisfy. The accuracy of the object location is specified by a set of points occurring within a predefined radius.

Finally, we present the hit-or-miss transformation where the use of a window is not necessary. As a consequence, the hit-or-miss transformation is more flexible and efficient to use in automated shape recognition.

\section{ACKNOWLEDGMENTS}

We thank the anonymous referees for their careful reading of a draft of this paper and the many insightful and critical comments which were seriously taken into consideration in the revision of the paper. 


\section{REFERENCES}

1. J. Serra, Image Analysis and Mathematical Morphology, Academic Press, New York, 1982.

2. G. Matheron, Random Set and Integral Geometry, Wiley, New York, 1975.

3. T. R. Crimmins and W. R. Brown, Image algebra and automatic shape recognition, IEEE Trans. Aerospace Electron. Systems, AES-21, Jan. 1985, 60-69.

4. C. R. Giardina and E. R. Dougherty, Morphological Methods in Image and Signal Processing, Prentice-Hall, Englewood Cliffs, NJ, 1987.

5. P. Maragos, A Unified Theory of Translation-Invariant Systems with Applications to Morphological Analysis and Coding of Irtages, Ph.D. thesis, Georgia Tech., Atlanta, GA, 1985.

6. P. Maragos, Tutorial on advances in morphological image processing and analysis, Opt. Engrg. 26, July 1987, 623-632.

7. R. M. Haralick, S. R. Sternberg, and X. Zhuang, Image analysis using mathematical morphology, IEEE Trans. Pattern Anal. Mach. Intell. PAMI-9, July 1987, 532-550.

8. P. Maragos and R. W. Schafer, Morphological filters-Part I: Their set-theoretic analysis and relations to linear shift invariant filters, IEEE Trans. ASSP ASSP-35, Aug. 1987, 1153-1169.

9. P. Maragos and R. W. Schafer, Morphological filters-Part II: Their relations to median, order-statistic, and stack filters, IEEE Trans. ASSP ASSP-35, Aug. 1987, 1170-1184.

10. P. Maragos, Pattern spectrum and multiscale shape recognition, IEEE Trans. Pattern Anal. Mach. Intell. PAMI-11, July 1989, 701716.

11. D. Sinha and C. R. Giardina, Discrete black and white object recognition via morphological functions, IEEE Trans. Pattern Anal. Mach. Intell. PAMI-12, Mar. 1990, 275-293.

12. X. Zhuang and R. M. Haralick, Morphological structuring element decomposition, Comput. Vision Graphics Image Process. 35, 1986, 370-382.

13. S. R. Sternberg, Grayscale morphology, Comput. Vision Graphics Image Process. 35, 1986, 333-355.

14. I. Pitas and A. N. Venetsanopoulos, Morphological shape decomposition, IEEE Trans. Pattern Anal. Mach. Intell. PAMI-12, Jan. 1990, 38-45.

15. F. Y. Shih and O. R. Mitchell, Automated fast recognition and location of arbitrarily shaped objects by image morphology, in Proc. Computer Vision and Pattern Recognition 88, pp. 774-779, Ann Arbor, MI, June 1988.

16. B. K. Jang and R. T. Chin, Analysis of thinning algorithms using mathematical morphology, IEEE Trans. Pattern Anal. Mach. Intell. PAMI-12, June 1990, 541-551.

17. D. G. Daut and D. Zhao, Mathematical morphology and its application in machine vision, in Visual Communications and Image Processing IV (1989), pp. 181-191, Philadelphia, PA, Nov. 1989.

18. E. Botha, J. Richards, and D. P. Casasent, Optical laboratory morphological inspection processor, Appl. Opt. 28, Dec. 1989, 53425350.

19. R. C. Vogt, Automatic generation of simple morphological algorithms, in Proc. Computer Vision and Pattern Recognition 88, pp. 760-765, Ann Arbor, MI, June 1988.
20. N. Ansari and E. Delp, Partial shape recognition: A landmarkbased approach, IEEE Trans. Pattern Anal. Mach. Intell. PAMI12. May 1990, 470-483.

21. P. K. Ghosh, A mathematical model for shape description using Minkowski operators, Comput. Vision Graphics Image Process. 44, 1989, 239-269.

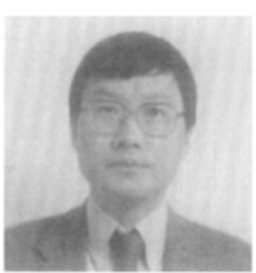

DONGMING ZHAO completed his undergraduate study in 1978 and received the M.S.E. in 1982 from Huazhong University of Science \& Technology, Wuhan, China, both in radio information engineering. $\mathrm{He}$ received the M.S.E. from the University of Michigan-Ann Arbor in 1983 and the Ph.D. from Rutgers University, New Brunswick, New Jersey, in 1990, both in electrical engineering. From 1987 to 1990 he was with the Machine Vision Group in the Center for Computer Aids for Industrial Productivity (CAIP) at Rutgers University. Since September 1990 he has been with the Department of Electrical and Computer Engineering at the University of Michigan-Dearborn as an assistant professor. His research interests include image analysis and machine vision, digital signal and image processing, image modcling and coding, and digital communications. Dr. Zhao is a member of the IEEE and its Computer Society and Signal Processing Society.

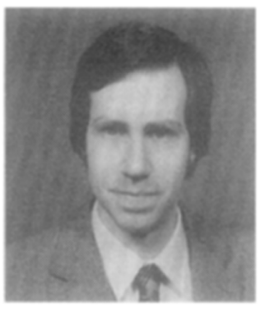

DAVID G. DAUT received the B.S. degree in electrical engineering from the New Jersey Institutc of Technology, Newark, in 1976, and the M.S. and Ph.D. degrees in electrical engineering from Rensselaer Polytechnic Institute, Troy, New York, in 1977 and 1981, respectively. From 1976 to 1980 he was a research assistant in the Department of Electrical, Computer and Systems Engineering, Rensselaer Polytechnic Institute, where his doctoral research had focused on image transmission in the over noisy communication channels. In 1980 he joined Rutgers University, Piscataway, New Jersey, as an assistant professor. Currently an associate professor in the Department of Electrical and Computer Engineering, he is responsible for teaching and research in the areas of communications and signal processing systems. His current research interests include image data compression techniques, digital communications, stochastic image modeling, and segmentation for realworld imagery analysis and machine vision. Dr. Daut is a member of the IECE, New York Academy of Sciences, the Association for Computing Machinery, and the Optical Society of America. 